\title{
Yukawa Coupling of Quantum Fields in Two Dimensions. I*
}

\author{
JaMes GLimm \\ Mass. Institute of Technology, Cambridge, Mass., USA
}

Received April 15, 1967

\begin{abstract}
A renormalization procedure is proposed. It gives rigorous mathematical meaning to the infinite cancellations in this model. A space cutoff is introduced in the interaction term $V$ and so $V$ has the form $\int_{|x| \leqq K} V(x) d x$, but there are no momentum cutoffs in $V$. There is an infinite constant and an infinite boson mass renormalization in this model. The main result is that the renormalized Hamiltonian is rigorously defined as a bilinear form in the Fock Hilbert space.
\end{abstract}

\section{Contents}

0. Introduction . . . . . . . . . . . . . . . . . . . . . . . . . . . 343

1. The Unrenormalized Hamiltonian and the Definition of $Q$. . . . . . . 348

2. The Dressing Transformation $T_{\varrho}$ and the Domain of the Renormalized

Hamiltonian . . . . . . . . . . . . . . . . . . . . . . . . . . 354

2.1. Introduction and Notation . . . . . . . . . . . . . . . . . . . 354

2.2. Properties of $T_{2 \varrho}$. . . . . . . . . . . . . . . . . . . . . . . . . . . . . . . . . . . . 355

2.3. Properties of $T_{1 \varrho}$. . . . . . . . . . . . . . . . . . . . . . . . . . . . . . . . . 357

2.4. The Basic Estimates . . . . . . . . . . . . . . . . . . . . . . 361

3. The Definition of $H_{\text {ren }}$ (Beginning) . . . . . . . . . . . . . . . . . . 368

3.1. Introduction . . . . . . . . . . . . . . . . . . . . . . . . . 368

3.2. Definition of $H_{1 \sigma}$. . . . . . . . . . . . . . . . . . . . . . . . . . . . . . . 369

3.3. Diagrams and Attached Products . . . . . . . . . . . . . . . . 370

3.4. Convergent Contributions to $H_{1}$. . . . . . . . . . . . . . . . . . . . 372

3.5. The Divergent Boson Self Energy Contribution to $H_{1}$. . . . . . . 375

3.6. The Self Energy as a Divergent Internal Line in Other Diagrams . . 379

3.7. The Definition of $H_{1}$. . . . . . . . . . . . . . . . . . . . . . 381

4. The Definition of $H_{\text {ren }}$ (Conclusion). . . . . . . . . . . . . . . . . . . 382

\section{Introduction}

We consider Quantum Fields interacting with Yukawa coupling in two dimensional space time. The interaction potential $V$ is restricted so that particles interact only when they lie in some bounded interval of space. But in all other respects the interaction is relativistic and does not contain momentum cutoffs. A renormalization procedure is proposed which gives rigorous mathematical meaning to the standard infinite

* This work was supported in part by the National Science Foundation, GP-6165. 
cancellations involved in defining the renormalized Hamiltonian. The renormalized Hamiltonian,

$$
H_{\text {ren }}=H_{\text {free }}+V+\text { (infinite terms) }
$$

is realized as a symmetric bilinear form densely defined in Fock space.

We split the interaction term $V$ into two parts,

$$
V=V_{1}+V_{2} \text {. }
$$

$V_{1}$ contains terms corresponding to fermion pair creation and fermion pair annihilation, while $V_{2}$ contains the remaining terms which correspond to the emission or absorption of a meson by a nucleon or antinucleon. We set

$$
\begin{gathered}
H_{1}=H_{\text {free }}+V_{1}+\text { (infinite terms) } \\
H_{\text {ren }}=H_{1}+V_{\mathbf{2}} .
\end{gathered}
$$

This definition is reasonable because in this model the only infinite renormalizations are the vacuum energy and the boson mass renormalizations, and both of these are due to terms from $V_{1}$ alone. We realize $H_{1}$ as a symmetric operator defined on a dense domain $\mathscr{D}_{H_{1}}$ contained in Fock space. $V_{2}$ and thus $H_{\text {ren }}$ are defined as symmetric bilinear forms on $\mathscr{D}_{H_{1}} \times \mathscr{D}_{H_{1}}$.

We show that $H_{1}$ and $H_{\text {ren }}$ are limits of operators $H_{1 \sigma}$ and $H_{\text {ren } \sigma}$,

$$
\begin{aligned}
H_{1} & =\lim H_{1 \sigma} \\
H_{\text {ren }} & =\lim H_{\text {ren } \sigma},
\end{aligned}
$$

in a sense which will be made precise later on. The cutoff operators, when expressed in momentum space, have all variables cut off at some $\sigma$, $\sigma \rightarrow \infty$. The renormalization terms in the cutoff operators are finite,depend on $\sigma$ and tend to infinity as $\sigma$ tends to infinity.

The interaction term is given formally by the singular expression

$$
V=\int: \Psi^{\dagger}(x) \Psi(x): \Phi(x) h(x) d x .
$$

Here $\Psi$ is the fermion field and $\Phi$ is the boson field, while $h$ is a cutoff function which limits the spatial extent of the interaction. Thus we assume $h$ is a smooth function which is identically one for $x$ in some bounded interval and which is identically zero for $x$ in the exterior of some larger bounded interval. The presence of this factor $h \neq 1$ means that the Schrödinger picture (in which we work) is not relativistic. We offer three reasons for including the space cutoff. First, all evidence indicates that a relativistic Schrödinger picture does not exist if one remains in the Fock Hilbert space. (As $h \rightarrow 1, \delta$ factors appear in our formulas or in perturbation theory. In the axiomatic approach, Haag's theorem is applicable if $h \equiv 1$, see [8, p. 161], [9, §6]). Secondly, our nonrelativistic Schrödinger picture can be used to construct (formally, at least) a 
relativistic Heisenberg picture. To show this, we write

$$
H_{\text {ren }}(h)=H_{\text {ren }}
$$

to show the dependence of $H_{\text {ren }}$ on $h$. Let $A$ be an observable associated with a bounded region of space time. Assume that the Schrödinger picture dynamics exist. That is, assume $H_{\text {ren }}(h)$ is a self adjoint operator. Take $h$ to be one on a large interval. Then

$$
A \rightarrow \exp \left(-i t H_{\text {ren }}(h)\right) A \exp \left(i t H_{\text {ren }}(h)\right)=A(t)
$$

defines an automorphism of the observables which is formally the correct relativistic dynamics in the Heisenberg picture, for $t$ suitably bounded.

In a subsequent paper we will show that the Schrödinger picture dynamics exist. However this result will require a finite and $h$-dependent mass renormalization in addition to the infinite renormalizations and thus we must be more careful in defining the automorphism (0.3). We work with an $h$ which is identically one on some interval $[-c, 1+c]$, where $c$ is the speed of light. This fixes the mass renormalization parameter $\delta m^{2}$. If $A$ is an observable associated with the interval (0.1) then (0.3) defines dynamics $A(t)$ which formally is relativisticly correct for $|t| \leqq 1$. If $A$ is an observable associated with an interval $(a, a+1)$ then $A(t)$ can be defined for $|t| \leqq 1$ by translations in space. An arbitrary observable $B$ is a limit of sums of products of observables $A_{i}$ associated with intervals $\left(a_{i}, a_{i}+1\right)$, and so it might be possible to define $B(t)$ for $|t| \leqq 1$ also, and this might yield the correct relativistic dynamics. If so, the process could be repeated and $B(t)$ could be defined for $|t| \leqq 2, \leqq 3, \ldots$ As a third reason, if one were willing to work in a framework larger than the Fock Hilbert space (cf. [1] and [5]), it is possible that some meaning could be given to the limit of our formulas as $h \rightarrow 1$.

The fact that we work in two dimensions instead of the physically correct four dimensions is more serious. This eliminates a second infinite mass renormalization (the fermion mass) and it eliminates an infinite charge renormalization.

We exhibit the domain $\mathscr{D}_{H_{1}}$ explicitly. We will construct an operator

$$
T=: \exp -\Gamma Q:
$$

with a domain $\mathscr{D}_{H_{1}}$ containing all Fock space vectors which

a) have at most a finite number of particles,

b) have compact support in momentum space.

In essence, we define

$$
\mathscr{D}_{H_{1}}=\text { range } T=T \mathscr{D}_{T} \text {. }
$$

There is a perturbation expansion for the correct choice of $Q$ [2]. We do not use the full expansion, which in any case probably does not converge. Instead we use certain terms from the first and second order con- 
tributions to this perturbation expansion. This is a sufficient number of terms so that the infinities present in the theory cancel exactly, but a small enough number of terms so that the infinite series defining $T$ converges. Thus we follow standard perturbation theory (in constructing $\mathscr{D}_{H_{1}}$ ) only far enough to obtain an exact cancellation of the infinities; once this is done we must obtain estimates to bound the remaining finite part (of $H_{1} \varphi, \varphi \in \mathscr{D}_{H_{1}}$, for example).

The mass renormalization parameter $\delta m^{2}$ appears to agree with perturbation theory to all arders in the coupling constant (modulo a finite renormalization) and the constant renormalization counter term we use appears to agree with perturbation theory at least up to second order.

We now try to motivate the formula (0.5). In the formal theory one finds a unitary equivalence between $H_{\text {ren }}$ and $H_{0}$,

$$
H_{\text {ren }} U=U H_{0} \text {. }
$$

In attempting a rigorous solution one should look for less. Our $T$ gives an approximate solution to (0.6). In fact we show that

$$
H_{1} T=T H_{0}+\text { finite terms . }
$$

The finite terms in this formula are rigorously defined unbounded operators. This formula, of course, leads to (0.5).

One thinks of states in the range of $T$ as representing physical particles. If $\Omega$ is the (free) vacuum state, then $T \Omega$ is an approximation to the physical vacuum. Now $V_{1}$ has the effect of creating and annihilating nucleon and antinucleon pairs, and this is responsible for the infinities of the theory. If $T \Omega$ is to approximate a physical vacuum, then $T \Omega$ is approximately an equilibrium state with respect to the creation and annihilation of pairs. This can happen only if $T \Omega$ contains a large number of nucleon antinucleon pairs. This phenomena is known as polarization of the vacuum. Since $\Omega$ is a state with no particles at all, $T$ must be an operator which creates a large number of nucleon antinucleon pairs. This will be the case provided the $Q$ in the definition of $T$ also creates pairs. We choose for $Q$ just the terms up to second order from perturbation theory which create pairs. We could have included more terms in $Q$, for example the terms up to second order which annihilate pairs. However it seems to cause essential difficulties if one attempts to put into $Q$ terms coming from both $V_{1}$ and $V_{2}$. In Sec. 3.1 and Sec. 3.3 we give simple formal arguments to show how our choice of $T$ permits us to identify and cancel the infinities in the theory.

We now discuss the convergence of $T$. The $\Gamma$ in $(0.4)$ is not an operator but an operation performed on the operator $Q . \Gamma$ is approximately an inverse to ad $H_{0}$, so

$$
\left[H_{0}, \Gamma Q\right] \approx Q .
$$


(We could work with an exact inverse to ad $H_{0}$, and did this in a preliminary version of this paper. However the exact inverse to ad $H_{0}$ presents technical difficulties which seem larger than its advantages.) Because $H_{0}$ is unbounded for momenta of large magnitude, $\Gamma Q$ is small relative to $Q$ as far as large values of momenta are concerned. This difference between $\Gamma Q$ and $Q$ is very important. $\Gamma Q$ is an unbounded operator with a dense domain. $Q$, however, is not an operator in the strict Hilbert space sense. It maps nonzero vectors into a function space larger than Hilbert space. $Q$ is essentially the operation of taking tensor products with some given function $\tilde{q}$, and $\tilde{q}$ is not in $L_{2}$. If $\varphi \in L_{2}, \varphi \neq 0$, then $\tilde{q} \otimes \varphi \notin L_{2} . \Gamma Q$ is essentially a tensor product operator also, but the associated function $\gamma \tilde{q}$ is in $L_{2}$.

In Sec. 2 we show that

$$
\left\|: \Gamma Q^{n}: \varphi\right\| \sim n ! K^{n}\|\varphi\| .
$$

This depends upon the fact that the interaction term $V$ is at most cubic and contains at least one fermion. The $n$ ! comes from the symmetrization laws. If we considered a general $V$ of order $j_{1}$ in the fermion fields and of order $j_{2}$ in the boson fields then the $n$ ! would appearently be replaced by

if $j_{1}<0$ and by

$$
(n !)^{\left(j_{l}-1+j_{2}\right) / 2}
$$

$$
(n !)^{j_{2} / 2}
$$

otherwise. Thus in some sense the first fermion does not count. We give in Sec. 2.4 some general estimates of this flavor. These estimates would probably be useful in studying other problems.

For

$$
T=\Sigma(n !)^{-1}:(-\Gamma Q)^{n}:
$$

we thus have the estimate

$$
\|T\| \sim\left(\Sigma K^{n}\right)\|\varphi\| .
$$

Here $K$ is large, in fact $K \rightarrow \infty$ as $h \rightarrow 1$. To deal with this, we remove from $Q$ and $\Gamma Q$ a part which contains only momenta of bounded magnitude. Call the remainders $Q_{\varrho}$ and $\Gamma Q_{\varrho}$. It is sufficient to work with $\Gamma Q_{\varrho}$ because the infinities are associated with the unbounded regions of momenta. Let $T_{\varrho}=: \exp \left(-\Gamma Q_{\varrho}\right):$. Our estimates give us

$$
\begin{gathered}
\left\|: T Q_{\varrho}{ }^{n}: \varphi\right\| \sim n ! K_{\varrho}{ }^{n}\|\varphi\| \\
\left\|T_{\varrho} \varphi\right\| \sim\left(\sum K_{\varrho}{ }^{n}\right)\|\varphi\|
\end{gathered}
$$

and $K_{\varrho} \rightarrow 0$ as $\varrho \rightarrow \infty$. We choose a fixed $\varrho$ large enough so that $K_{\varrho}<1$, and we replace $T$ in (0.5) by $T_{\varrho}$.

The author's understanding of this subject has been aided by conversations with a number of people, including K. SymanziK, P. Kristensen, D. KastLer and D. RUELLE. We thank W. FARIS for finding a number of minor errors and misprints. 


\section{The Unrenormalized Hamiltonian and the Definition of $Q$}

The Lorentz group in two dimensions is much more elementary than the Lorentz group in four or three dimensions. The homogeneous Lorentz is one dimensional and its identity component consists of the transformations

$$
\Lambda(\alpha)=\left(\begin{array}{cc}
\cosh \alpha & \sinh \alpha \\
\sinh \alpha & \cosh \alpha
\end{array}\right) .
$$

This leads to notable simplifications in notation. (It may also lead to simplifications of the mathematical difficulties, cf. [9, p. 34], but we have not made use of this.) In particular the Dirac equation in two dimensions can be replaced by a scalar valued Klein Gordon equation. Thus we construct three Hilbert spaces

$$
\mathbf{D}^{+}, \mathbf{D}^{+\prime} \text {, and } \mathbf{K}^{+}
$$

consisting of scalar valued functions of a single real variable $k$ or $p$, with the inner products

where

$$
\begin{aligned}
(f, g)_{D}=(f, g)_{D^{\prime}} & =\int \bar{f}(p) g(p) \omega(p)^{-1} d p \\
(f, g)_{K} & =\int \bar{f}(k) g(k) \mu(k)^{-1} d k,
\end{aligned}
$$

$$
\begin{aligned}
& \omega(p)=\left(\omega_{0}^{2}+p^{2}\right)^{1 / 2} \\
& \mu(k)=\left(\mu_{0}^{2}+k^{2}\right)^{1 / 2}
\end{aligned}
$$

and $\omega_{0}, \mu_{0}>0$. The spaces $\mathbf{D}^{+}$and $\mathbf{D}^{+\prime}$ are respectively the spaces of single particle nucleons and antinucleons, while $\mathbf{K}^{+}$consists of the single particle meson wave functions. The Fock space for our problem is the tensor product

$$
\mathbf{F}=G\left(\mathbf{D}^{+} \oplus \mathbf{D}^{+\prime}\right) \otimes S\left(\mathbf{K}^{+}\right) .
$$

Here $G\left(\mathbf{D}^{+} \oplus \mathbf{D}^{+\prime}\right)$ is the Grassmann algebra over $\mathbf{D}^{+} \oplus \mathbf{D}^{+\prime}$, that is the direct sum of alternating tensors of all orders from $\mathbf{D}^{+} \oplus \mathbf{D}^{+\prime}$. Similarly $S$ denotes a symmetric algebra, or the direct sum of symmetric tensors of all orders. We introduce the annihilation operators

$$
b(p), b^{\prime}(p), a(k)
$$

and their adjoints, the creation operators

$$
b^{*}(p), b^{*}(p), a^{*}(k)
$$

which annihilate and create nucleons, antinucleons and mesons respectively. These are normalized so that

and

$$
\left\{b(p), b^{*}(q)\right\}=\left\{b^{\prime}(p), b^{*}(q)\right\}=\delta(p-q)
$$

$$
\left[a(k), a^{*}(l)\right]=\delta(k-l) .
$$


Let $\varphi_{m}$ be a state in $\mathbf{F}$ with exactly $m$ mesons. If we write $\varphi_{m}=\varphi_{m}\left(k_{1}, \ldots, k_{m}\right)$ as a function of its meson variables (and we suppress the fermion variables) then $a$ and $a^{*}$ are defined formally by the formulas

$$
\begin{aligned}
& \quad\left(a(k) \varphi_{m}\right)\left(k_{1}, \ldots, k_{m-1}\right)=\left(\mu(k)^{-1} m\right)^{1 / 2} \varphi_{m}\left(k_{1}, \ldots, k_{m-1}, k\right) \\
& \left(a^{*}(k) \varphi_{m}\right)\left(k_{1}, \ldots, k_{m+1}\right) \\
& =\left(\mu(k)(m+1)^{-1}\right)^{1 / 2} \sum_{j} \delta\left(k-k_{j}\right) \varphi_{m}\left(k_{1}, \ldots, k_{j-1}, k_{j+1}, \ldots, k_{m+1}\right) .
\end{aligned}
$$

To obtain a similar expression for the $b$ 's, it is convenient to distinguish between the nucleon and antinucleon variables. To do this we introduce a variable $\varepsilon$ which takes on the value \pm 1 only. We associate +1 with nucleons and -1 with antinucleons (so that $\varepsilon$ represents nucleon,, charge"). We denote by

$$
\varphi_{1}(p, \varepsilon) \in\left(\mathbf{D}^{+} \oplus \mathbf{D}^{+\prime}\right) \otimes S\left(\mathbf{K}^{+}\right)
$$

a single fermion state, where

$$
\begin{aligned}
& \varphi_{1}(p,+1) \in \mathbf{D}^{+} \otimes S\left(\mathbf{K}^{+}\right) \\
& \varphi_{1}(p,-1) \in \mathbf{D}^{+\prime} \otimes S\left(\mathbf{K}^{+}\right)
\end{aligned}
$$

are the projections of $\varphi_{\mathbf{1}}$ onto $\mathbf{D}^{+} \otimes S\left(\mathbf{K}^{+}\right)$and $\mathbf{D}^{+\prime} \otimes S\left(\mathbf{K}^{+}\right)$respectively. Let $\varphi$ be a state in $\mathbf{F}$ with exactly $n$ fermions. We can write

$$
\varphi=\varphi\left(p_{1}, \varepsilon_{1}, \ldots, p_{n}, \varepsilon_{n}\right)
$$

if (as above) we suppress the meson variables. Then $b$ and $b^{*}$ are defined formally by

$$
\begin{aligned}
& (b(p) \varphi)\left(p_{1}, \varepsilon_{1}, \ldots, p_{n-1}, \varepsilon_{n-1}\right) \\
& \quad=\left(\omega(p)^{-1} n\right)^{1 / 2} \varphi\left(p_{1}, \varepsilon_{1}, \ldots, p_{n-1}, \varepsilon_{n-1}, p,+1\right) \\
& \begin{array}{r}
\left(b^{*}(p) \varphi\right)\left(p_{1}, \varepsilon_{1}, \ldots, p_{n+1}, \varepsilon_{n+1}\right) \\
=\left(\omega(p)(n+1)^{-1}\right)^{1 / 2} \sum_{j}(-1)^{j-n-1} \delta\left(p-p_{j}\right) \delta\left(1-\varepsilon_{j}\right) \times \\
\quad \times \varphi\left(p_{1}, \varepsilon_{1}, \ldots, p_{j-1}, \varepsilon_{j-1}, p_{j+1}, \varepsilon_{j+1}, \ldots\right) .
\end{array}
\end{aligned}
$$

The expressions for $b^{\prime}$ and $b^{*}$ are similar and are given by replacing +1 by -1 . The meson field is given by (cf. [4, p. 102])

$$
\begin{aligned}
\Phi(x)=\int e^{i\left(k x^{1}-\mu(k) x^{0}\right)} a(k) \mu(k)^{-1 / 2} d k & + \\
& +\int e^{-i\left(k x^{1}-\mu(k) x^{0}\right)} a^{*}(k) \mu(k)^{-1 / 2} d k .
\end{aligned}
$$

According to well known ideas, this expression is a distribution in the variable $x$ and $\int \Phi(x) f(x) d x$ has meaning as an unbounded operator in Fock space $\mathbf{F}$. If one admits a wider framework than Fock space, then $\Phi(x)$ is meaningful for each $x$. The fermion (nucleon-antinucleon) field $\Psi(x)$ is slightly more complicated. It has two components,

$$
\Psi(x)=\Psi^{1}(x), \Psi^{2}(x),
$$


and in contrast to $\Phi$, does not transform as a scalar under Lorentz transformations. In the single particle spaces we introduce the representation

$$
\begin{aligned}
& \Lambda(\alpha): f(p) \rightarrow f(p \cosh \alpha-\omega(p) \sinh \alpha) \\
& \Lambda(\alpha): f(k) \rightarrow f(k \cosh \alpha-\mu(k) \sinh \alpha) .
\end{aligned}
$$

There is a unitary representation $U(\Lambda)$ of the connected homogeneous Lorentz group in F. It is simply the direct sum of tensor products of the above single particle representations. The field $\Psi(x)$ must satisfy the transformation law ([4, p. 163, p. 305])

$$
\left(\begin{array}{cc}
e^{-\alpha / 2} & 0 \\
0 & e^{\alpha / 2}
\end{array}\right) \begin{aligned}
& \Psi^{1}(\Lambda(\alpha) x) \\
& \Psi^{2}(\Lambda(\alpha) x)
\end{aligned}=U(\Lambda(\alpha)) \Psi(x) U^{-1}(\Lambda(\alpha)) .
$$

We have the formulas

$$
\begin{aligned}
\Psi^{1}(x) & =\int e^{i\left(p x^{1}-\omega(p) x^{0}\right)}(\omega(p)+p)^{1 / 2} \omega(p)^{-1 / 2} b(p) d p+ \\
& +\int e^{-i\left(p x^{1}-\omega(p) x^{0}\right)}(\omega(p)+p)^{1 / 2} \omega(p)^{-1 / 2} b^{\prime *}(p) d p \\
\Psi^{2}(x) & =\int e^{i\left(p x^{1}-\omega(p) x^{0}\right)}(\omega(p)-p)^{1 / 2} \omega(p)^{-1 / 2} b(p) d p- \\
& -\int e^{-i\left(p x^{1}-\omega(p) x^{0}\right)}(\omega(p)-p)^{1 / 2} \omega(p)^{-1 / 2} b^{*}(p) d p .
\end{aligned}
$$

For the relation between this expression for $\Psi$ and the conventional $\Psi$ as an operator on spinor valued functions, see the appendix. We define the adjoint fermion field by the formula

$$
\Psi^{\dagger}(x)=\Psi^{2}(x)^{*}, \quad \Psi^{1}(x)^{*} .
$$

The inner product over the indices $i=1,2$ of $\Psi^{\dagger}$ and $\Psi$ is

$$
\Psi^{\dagger} \Psi(x)=\Psi^{2}(x)^{*} \Psi^{1}(x)+\Psi^{1}(x)^{*} \Psi^{2}(x) .
$$

Finally $V$ is given by the Wick ordered integral

$$
V=\int_{x_{0}=0}: \Psi^{\dagger} \Psi(x): \Phi(x) h(x) d x^{1} .
$$

On Fock space this is a formal expression but not an operator. The free Hamiltonian is

$$
\begin{aligned}
H_{0} & =\int\left[b^{*}(p) \omega(p) b(p)+b^{\prime *}(p) \omega(p) b^{\prime}(p)\right] d p+ \\
& +\int a^{*}(k) \mu(k) a(k) d k \\
& =H_{0_{f}}+H_{0_{b}},
\end{aligned}
$$

and the unrenormalized Hamiltonian is $H=H_{0}+V$. For simplicity we have set the coupling constant $g=1$. Our results are valid for any value of $g$.

Each of the fields $\Psi$ and $\Phi$ entering into the definition of $V$ can be expressed as a sum of its positive and negative frequency components, and this gives rise to a decomposition of $V$ into a sum of eight terms. Two of these terms, those corresponding to fermion pair creation, are 
present in $Q$. In particular we let

$$
\begin{aligned}
& q_{1}\left(p_{1}, p_{2}, k\right)=\hat{h}\left(p_{1}+p_{2}+k\right) a^{*}(k) b^{*}\left(p_{1}\right) b^{* *}\left(p_{2}\right) \mu^{-1 / 2} S\left(p_{1}, p_{2}\right) \\
& q_{2}\left(p_{1}, p_{2}, k\right)=\hat{h}\left(p_{1}+p_{2}-k\right) a(k) b^{*}\left(p_{1}\right) b^{\prime *}\left(p_{2}\right) \mu^{-1 / 2} S\left(p_{1}, p_{2}\right)
\end{aligned}
$$

where $\hat{h}$ is the Fourier transform of $h, \omega_{i}=\omega\left(p_{i}\right)$ and

$$
\begin{gathered}
S\left(p_{1}, p_{2}\right)=\left(\omega_{1} \omega_{2}\right)^{-1 / 2}\left[\left(\omega_{1}-p_{1}\right)^{1 / 2}\left(\omega_{2}+p_{2}\right)^{1 / 2}-\right. \\
\left.-\left(\omega_{1}+p_{1}\right)^{1 / 2}\left(\omega_{2}-p_{2}\right)^{1 / 2}\right] \\
=-\left(\omega_{1} \omega_{2}\right)^{-1 / 2} 2^{1 / 2}\left(\omega_{1} \omega_{2}-p_{1} p_{2}-\omega_{0}^{2}\right)^{1 / 2} \operatorname{sgn}\left(p_{1}-p_{2}\right) .
\end{gathered}
$$

Then the (formal) operators

and

$$
Q_{1}=\int q_{1} d p_{1} d p_{2} d k
$$

$$
Q_{2}=\int q_{2} d p_{1} d p_{2} d k
$$

are these two terms from $V$. Whenever we have an expression such as (1.3) or (1.4), we will call the integrand $q_{i}$ the operator kernel of $Q_{i}$. It will also be convenient to introduce the numerical kernel.

$$
\tilde{q}_{i}=\hat{h}\left(p_{1}+p_{2}+k\right) \mu^{-1 / 2} S\left(p_{1}, p_{2}\right) .
$$

Thus the operator kernel $q_{i}$ is just the numerical kernel $\tilde{q}_{i}$ times the appropriate annihilation and creation operators.

Following Friedrichs [2] we define

$$
\Gamma Q_{1}=\int\left(\omega_{1}+\omega_{2}+\mu\right)^{-1} q_{1} d p_{1} d p_{2} d k .
$$

Because of the decrease at infinity, $\Gamma Q_{1}$ is better behaved than $Q_{1}$ and we will see without difficulty that $\Gamma Q_{1}$ is an unbounded densely defined operator. Let

$$
\begin{gathered}
\Gamma Q_{2}=\int\left(\omega_{1}+\omega_{2}-\mu-i\right)^{-1} q_{2} d p_{1} d p_{2} d k \\
Q_{3}=-Q_{2} \Gamma Q_{1}+: Q_{2} \Gamma Q_{1} \\
=-Q_{2}-O-\Gamma Q_{1} .
\end{gathered}
$$

The expression $Q_{2}-0-\Gamma Q_{1}$ is called the attached product and is defined by the equation above, see [2].

We now define the Wick product indicated by the double colon in (1.5). A Wick product

$$
: R_{1} \ldots R_{n}:
$$

is multilinear in the factor $R_{1}, \ldots, R_{n}$. Thus it is sufficient to define the Wick product

$$
: a\left(k_{1}\right) \ldots b^{*}\left(p_{m}\right):
$$

This Wick product is just plus or minus the ordinary product of the same operators, but arranged in such an order that all the creators are at the left and all the annihilators are located to the right. The minus is chosen if there is an odd number of interchanges of adjacent fermion operators required to achieve this new order. Otherwise the plus is chosen. 
The formal operator $Q_{3}$ contains four fermion creators, and so it can be expressed by an integral similar to (1.3) and (1.4). That is, there is an operator kernel $q_{3}$,

and

$$
q_{3}=\tilde{q}_{3}\left(p_{1}, p_{2}, p_{3}, p_{4}\right) b^{*}\left(p_{1}\right) b^{\prime *}\left(p_{2}\right) b^{*}\left(p_{3}\right) b^{*}\left(p_{4}\right)
$$

Let

$$
Q_{3}=\int q_{3} d p_{1} d p_{2} d p_{3} d p_{4} .
$$

$$
\Gamma Q_{3}=\int\left(\omega_{1}+\omega_{2}+\omega_{3}+\omega_{4}\right)^{-1} q_{3} d p_{1} d p_{2} d p_{3} d p_{4} .
$$

Finally we define

$$
\begin{aligned}
Q & =Q_{1}+Q_{2}+Q_{3} \\
\Gamma Q & =\Gamma Q_{1}+\Gamma Q_{2}+\Gamma Q_{3} .
\end{aligned}
$$

$\Gamma$ is an approximate inverse to ad $H_{0}$. In other words formally we have

$$
\begin{gathered}
{\left[H_{0}, \Gamma Q_{1}\right]=Q_{i}, \quad i=1 \text { or } 3} \\
{\left[H_{0}, \Gamma Q_{2}\right]=Q_{2}+\int i\left(\omega_{1}+\omega_{2}-\mu-i\right)^{-1} q_{2} d p_{1} d p_{2} d k .}
\end{gathered}
$$

We have the following explicit formula for the numerical kernel $\tilde{q}_{3}$ in (1.5)

$$
\tilde{q}_{3}=\int \tilde{q}_{2}\left(p_{1}, p_{2}, k\right) \tilde{q}_{1}\left(p_{3}, p_{4}, k\right)\left(\omega_{3}+\omega_{4}+\mu\right)^{-1} d k .
$$

Observe that $\tilde{q}_{3}$ is not antisymmetrical in its four variables and that there is a decrease at infinity in the last two variables which does not occur in the first two variables.

We now show that $\Gamma Q$ is an operator. The next lemma is known and is not hard to prove, starting from the definition of the annihilation and creaton operators.

Lemma 1.1. Let $r(p, k)$ be an $L_{2}$ function of $j$ variables and let

$$
R=\int \tilde{r} b^{*}\left(p_{1}\right) \ldots b^{\prime}\left(p_{j_{1}}\right) a^{*}\left(k_{1}\right) \ldots a\left(k_{2}\right) d p d k
$$

be an operator with a numerical kernel $\tilde{r}$. Then

$$
\|R\| \leqq \text { const. }\|\tilde{r}\|_{2}\left\|(N+I)^{j} \varphi\right\|
$$

for any state $\varphi$ in the domain of $(N+I)^{j}$, and the constant depends only on $j$.

In this lemma, $N$ is the number of particles operator and we assume that the operators in the integrand of (1.7) are Wick ordered.

Lemma 1.2. Suppose $0 \leqq \tau<2^{-1}$. The following functions are in $L_{2}$.

$$
\begin{gathered}
\left(\omega_{1}^{\tau}+\omega_{2}^{\tau}+\mu^{\tau}\right)\left(\omega_{1}+\omega_{2}+\mu\right)^{-1} \tilde{q}_{1} \\
\left(\Sigma \omega_{i}^{\tau}\right)\left(\Sigma \omega_{i}\right)^{-1} \tilde{q}_{3} \\
\left(\sum_{i \neq j} \omega_{i}^{\tau} \omega_{j}^{\tau}-\omega_{1}^{\tau} \omega_{2}^{\tau}\right)\left(\Sigma \omega_{i}\right)^{-1} \tilde{q}_{3} .
\end{gathered}
$$


Also the function

$$
\left(\omega_{1}^{\tau}+\omega_{2}^{\tau}\right)\left(\omega_{1}+\omega_{2}-\mu-i\right)^{-1} \tilde{q}_{2}
$$

is in $L_{2}$ on any set in which the $k$ variable of $\tilde{q}_{2}$ is bounded.

From this lemma we see that $\Gamma Q$ is an operator and furthermore that

$$
\begin{aligned}
& R(1, \tau)=\int\left(\omega_{1}^{\tau}+\omega_{2}^{\tau}+\mu^{\tau}\right)\left(\omega_{1}+\omega_{2}+\mu\right)^{-1} q_{1} d p d k \\
& R(2, \tau)=\int\left(\omega_{1}^{\tau}+\omega_{2}^{\tau}\right)\left(\omega_{1}+\omega_{2}-\mu-i\right)^{-1} q_{2} d p d k \\
& R(3, \tau)=\int\left(\Sigma \omega_{i}^{\tau}\right)\left(\Sigma \omega_{i}\right)^{-1} q_{3} d p
\end{aligned}
$$

are operators for $0 \leqq \tau<2^{-1}$.

Proof of Lemma 1.2. The function $\hat{h}$ is rapidly decreasing at infinity because $h$ is smooth. We set

Then (1.8) is bounded by

$$
\eta=p_{1}+p_{2}, \quad \xi=p_{1}-p_{2} .
$$

$\left(\omega_{1}+\omega_{2}+\mu\right)^{-(1-\tau)}\left|\tilde{q}_{1}\right| \leqq$ const. $(|\xi|+1)^{-(1 / 2)-\gamma} \mu^{-(1 / 2)-\gamma}(|\eta+k|+1)^{-1}$ for some positive $\gamma$ depending on $\tau$. The constant depends only on $h$, and we see that (1.8) is in $L_{\mathbf{2}}$. The same reasoning implies

$$
\begin{aligned}
& \left(\omega_{1}^{\tau}+\omega_{2}^{\tau}\right)\left(\omega_{1}+\omega_{2}\right)^{-1}\left|\tilde{q}_{2}\right| \leqq\left(\omega_{1}+\omega_{2}\right)^{-(1-\tau)}\left|\tilde{q}_{2}\right| \leqq \\
& \leqq \text { const. }(|\xi|+1)^{-(1 / 2)-\gamma} \mu^{-1 / 2}(|\eta|+1)^{-\gamma}(|\eta+k|+1)^{-1},
\end{aligned}
$$

so (1.12) is in $L_{2}$. For $k$ restricted to a bounded set, this shows that (1.11) is in $L_{2}$.

To show that (1.10) is in $L_{2}$ we have to bound

$$
\omega_{j_{1}}^{\tau} \omega_{j_{2}}^{\tau}\left(\sum_{i} \omega_{i}\right)^{-1} \tilde{q}_{3}
$$

where $j_{2}=3$ or $j_{2}=4$. This function is bounded by

$$
\int\left(\omega_{1}+\omega_{2}\right)^{-(1-\tau)}\left|\tilde{q}_{2}\right|\left(\omega_{1}+\omega_{2}+\mu\right)^{-(1-\tau)}\left|\tilde{q}_{1}\right| d k,
$$

which is in $L_{2}$ by our estimates above. Since $\omega_{j}^{\tau}$ is bounded away from zero, we also conclude that (1.9) is in $L_{2}$.

In order to deal rigorously with the (formal) operators $Q$ and $Q_{i}$ we introduce cutoff versions of these operators. The cutoff operators will have dense domains.

Let $\sigma=\left(\sigma_{b}, \sigma_{f}\right)$ be a pair of positive numbers. If $b^{\#}$ is a fermion annihilation or creation operator we define the cutoff operator $b_{\sigma}^{\#}$ by the formula

$$
b_{\sigma}^{\#}(p)=\left\{\begin{array}{l}
b^{\#}(p) \text { if }|p| \leqq \sigma_{f} \\
0 \text { otherwise } .
\end{array}\right.
$$

We define the cutoff meson operators $a_{\sigma}(k)$ and $a_{\sigma}^{*}(k)$ similarly. Given an operator $Q_{1}$, etc. depending on $b^{*}(p)$, etc., we define $Q_{1 \sigma}$ to be the operator obtained by substituting $b_{\sigma}^{*}(p)$ for $b^{*}(p)$, etc. In order to obtain convergence of our series for $T$ (even for large values of the coupling constant) 
we also introduce the lower cutoff

$$
\begin{aligned}
& Q_{1 \varrho}=\int_{\left|p_{j}\right| \leqq \varrho} q_{1} d p_{1} d p_{2} d k \\
& Q_{2 \varrho}=\int_{\left|p_{j}\right| \leqq \varrho} q_{2} d p_{1} d p_{2} d k \\
& Q_{3 \varrho}=\int_{\left|p_{j}\right| \leqq \varrho} q_{3} d p,
\end{aligned}
$$

and we consider operators $Q_{1 \varrho \sigma}$, etc. with a double cutoff. In the course of our proofs we choose a large fixed value of $\varrho$ and we let $\sigma \rightarrow \infty$.

\section{The Dressing Transformation $\boldsymbol{T}_{e}$ and the Domain of the Renormalized}

\section{Hamiltonian}

\subsection{Introduction and Notation}

In Sec. 2 we construct explicitly the domain $\mathscr{D}_{H_{1}}$. In Sec. 3 and Sec. 4 the renormalized Hamiltonian will be realized as a bilinear form on $\mathscr{D}_{H_{1}} \times \mathscr{D}_{H_{1}}$. We prove in Sec. 2 that $\mathscr{D}_{H_{1}}$ is a dense subset of Fock space (Theorem 2.3.3). Of course the most significant part of this is that $\mathscr{D}_{H_{1}}$ is contained in Fock space (consists of normalized vectors) or in other words that the power series defining the dressing transformation $T_{\varrho}$ converges (Theorem 2.2.1 and 2.3.1).

Let $D(n, K)$ be the set of all Fock space vectors which

a) have at most $n_{1}$ fermions and at most $n_{2}$ bosons, $n_{1}+3 n_{2} \leqq n$,

b) are equal to zero whenever at least one of the bosons has a momentum $k_{j}$ with magnitude larger than $K$.

Let

$$
\begin{aligned}
& T_{Q}=: \exp \left(-\Gamma Q_{\varrho}\right): \\
& \mathscr{D}_{T^{\prime}}=\cup_{n, K} D(n, K) .
\end{aligned}
$$

We prove that for sufficiently large $\varrho$, the series for

$$
T_{Q} \varphi, \quad \varphi \in \mathscr{D}_{T}
$$

necessarily converges to an element of $\mathbf{F}$. We will pick a suitable such $\varrho$ and then we define

$$
\begin{gathered}
\mathscr{D}_{H_{1}}=T_{\varrho}\left(\mathscr{D}_{T} \cap \mathscr{D}_{H_{0}^{2}}\right) \\
=\left\{T_{\varrho} \varphi: \varphi \in \mathscr{D}_{T} \text { and }\left\|H_{0}^{2} \varphi\right\|<\infty\right\} .
\end{gathered}
$$

The Wick exponential $T$ factors into a product of two ordinary exponentials,

$$
\begin{aligned}
T & =T_{1} T_{2} \\
T_{1} & =\exp \left(-\Gamma Q_{1}-\Gamma Q_{3}\right) \\
T_{2} & =\exp \left(-\Gamma Q_{2}\right)
\end{aligned}
$$


and $T_{\varrho}$ and $T_{\varrho \sigma}$ factor similarly. Only the first factor presents difficulties and we treat it separately. The infinite series defining $T_{2}$ breaks off after a finite number of terms, and thus is trivial.

For $0 \leqq \tau<1$, let

$$
\begin{gathered}
F_{\tau}=F(\tau)=\int a^{*}(k) \mu^{\tau} a(k) d k+ \\
+\int\left[b^{*}(p) b(p)+b^{*}(p) b^{\prime}(p)\right] \omega^{\tau} d p .
\end{gathered}
$$

Thus $F_{0}=N$ is the number of particles operator and as $\tau \rightarrow 1, F_{\tau} \rightarrow H_{0}$. If $\varphi \in \mathbf{F}$ let $\varphi_{n}$ be the $n$ particle component of $\varphi$. It will be convenient to introduce the following subspaces of $\mathbf{F}$, depending on $\lambda>0$ (cf. [6]):

$$
\begin{gathered}
\mathscr{E}(\lambda)=\mathscr{D}_{\exp (\lambda N)}=\left\{\varphi: \sum_{n}\left\|e^{\lambda n} \varphi_{n}\right\|^{2}<\infty\right\} \\
\mathscr{F}(\lambda, \tau)=\mathscr{D}_{F(\tau) \exp (\lambda N)}=\left\{\varphi: \sum_{n}\left\|e^{\lambda n} F_{\tau} \varphi_{n}\right\|^{2}<\infty\right\} .
\end{gathered}
$$

\subsection{Properties of $T_{2 \varrho}$}

Theorem 2.2.1. The operators $T_{2}, T_{2 \varrho}$ and $T_{2 \varrho \sigma}$ are invertible maps of $\mathscr{D}_{T}$ onto $\mathscr{D}_{T^{\prime}}$. They leave each subspace $\bar{D}(n, K)$ invariant and map

$$
D(n, K) \cap \mathscr{D}_{H_{0}^{2}}
$$

onto a subspace of $D(n, K)$ which is dense in $D(n, K)$ in the $\mathbf{F}$ norm. Also

$$
\begin{array}{lll}
\lim _{\sigma \rightarrow \infty} T_{2 \varrho \sigma} \varphi=T_{2 \varrho} \varphi, & & \varphi \in \mathscr{D}_{T} . \\
\lim _{\sigma \rightarrow \infty} T_{2 \sigma} \varphi=T_{2} \varphi, & & \varphi \in \mathscr{D}_{T} .
\end{array}
$$

Proof. Let $K$ be given. In order to study

$$
\Gamma Q_{2} \varphi, \varphi \in D(n, K),
$$

we can limit the $k$ integration in the definition of $\Gamma Q_{2} \varphi$ to the interval $[-K, K]$. This changes $\Gamma Q_{2}$ but does not affect the product $\Gamma Q_{2} \varphi$. However the new operator has the form of the $R$ of Lemma 1.1 and the kernel is in $L_{2}$ by Lemma 1.2. We conclude that $\Gamma Q_{2} \varphi$ is in $\mathbf{F}$ and it is easy to see that it is also in $D(n-1, K)$. It follows that the power series for

$$
\exp \left( \pm \Gamma Q_{2}\right) \varphi, \quad \varphi \in \mathscr{D}_{T}
$$

terminates after a finite number of terms and $T_{2}^{-1}=\exp \left(\Gamma Q_{2}\right)$. This proves the first statement.

Let $\theta \in D(n, K)$ and let $\left(T_{2}^{-1} \theta\right)_{\lambda}$ be a sequence in $D(n, K) \cap \mathscr{D}_{H_{0}^{2}}$ converging to $T_{2}^{-1} \theta$. From Lemma 1.1 we see that $\Gamma Q_{2}$ and $T_{2}$ are continuous as operators from $D(n, K)$ into $\mathbf{F}$. Thus

$$
\theta=T_{2} T_{2}^{-1} \theta=\lim _{\lambda} T_{2}\left(T_{2}^{-1} \theta\right)_{\lambda}
$$

The limit statements in the theorem also follow from Lemma 1.1. 
The proof of Theorem 2.2.1 also gives us

Lemma 2.2.1. Let $0 \leqq \tau<2^{-1}$. Then

$$
\begin{gathered}
R(2, \tau), \quad R(2, \tau)_{\varrho}, \quad R(2, \tau)_{\varrho \sigma} \\
{\left[H_{0}, \Gamma Q_{2 \varrho}\right]-Q_{2 \varrho}, \quad\left[H_{0}, \Gamma Q_{2 \varrho \sigma}\right]-Q_{2 \varrho \sigma}}
\end{gathered}
$$

define bounded operators from $D(n, K)$ into $\mathbf{F}$. Also

$$
\begin{gathered}
\lim _{\sigma \rightarrow \infty}\left(R(2, \tau)_{\varrho}-R(2, \tau)_{\varrho \sigma}\right) \varphi=0 \\
\lim _{\sigma \rightarrow \infty}\left(\left[H_{0}, \Gamma Q_{2 \varrho}-\Gamma Q_{2 \varrho \sigma}\right]-Q_{2 \varrho}+Q_{2 \varrho \sigma}\right) \varphi=0
\end{gathered}
$$

for $\varphi$ in $\mathscr{D}_{T}$ and the convergence is uniform if $\varphi$ lies in a bounded set in $D(n, K)$.

This lemma is typical of a sequence of lemmas that we will prove in Sec. 3. They will concern operators

and

$$
R=\int r(p, k) c(p, k) d p d k
$$

$$
R(\sigma)=\int r(p, k, \sigma) c(p, k) d p d k
$$

where $c$ is a Wick ordered product of creation and annihilation operators as in (1.7). We will prove

a) $R\left(H_{0}+I\right)^{-2}$ and $R(\sigma)\left(H_{0}+I\right)^{-2}$ define bounded operators from $D(n, K)$ into $\mathbf{F}$.

b) $\lim (R-R(\sigma)) \varphi=0$ for each $\varphi$ in $\mathscr{D}_{T} \cap \mathscr{D}_{H_{0}^{2}}$ and the convergence is uniform in $\varphi$ 's for which the vector $\left(H_{0}+I\right)^{2} \varphi$ lies in a bounded set in $D(n, K)$.

Theorem 2.2.2. Let $R$ and $R(\sigma)$ be given as above and suppose $a$ ) and $b$ ) Then

$\left.\mathrm{a}^{\prime}\right): T_{2 \varrho} R:$ and $: T_{2 \varrho \sigma} R(\sigma):$ are defined as operators from $\mathscr{D}_{T} \cap \mathscr{D}_{H_{0}^{2}}$ into $\mathbf{F}$.

$\left.\mathrm{b}^{\prime}\right)$ The limit

$$
\left(: T_{2 \varrho} R-T_{2 \varrho \sigma} R(\sigma):\right) \varphi \rightarrow 0
$$

holds for each $\varphi$ in $\mathscr{D}_{T} \cap \mathscr{D}_{H_{0}^{2}}$.

If we assume a) but not b) then the conclusion $a^{\prime}$ ) is still valid.

Proof. It is sufficient to consider $: \Gamma Q_{2 \varrho}{ }^{m} R$ : in place of $: T_{2 \varrho} R:$. Let $\varphi$ be a state in $D(n, K) \cap \mathscr{D}_{H_{0}^{2}}$ with a definite number of mesons. Let $k^{\prime \prime}$ denote the last $m$ meson variables and let $k^{\prime}$ denote the other meson variables in $\varphi$. If we hold $k^{\prime \prime}$ fixed and regard $\varphi$ as a function of $k^{\prime}$ and the fermion variables, the result is a state $\theta\left(k^{\prime \prime}\right)$ in $D(n, K) \cap \mathscr{D}_{H_{0}^{2}}$ depending measurably on a parameter $k^{\prime \prime}$. By hypothesis $R \theta=(R \theta)\left(k^{\prime \prime}\right)$ and $R(\sigma) \theta$ are defined; they depend measurably on $k^{\prime \prime}$. Moreover $\int\left\|(R \theta)\left(k^{\prime \prime}\right)\right\|^{2} d k^{\prime \prime} \leqq c \int\left\|\left(H_{0}+I\right)^{2} \theta\left(k^{\prime \prime}\right)\right\|^{2} d k^{\prime \prime} \leqq c\left\|\left(H_{0}+I\right)^{2} \varphi\right\|^{2}(2.2 .1)$ and

$$
\begin{gathered}
\int\left\|(R(\sigma) \theta)\left(k^{\prime \prime}\right)-(R \theta)\left(k^{\prime \prime}\right)\right\|^{2} d k^{\prime \prime} \leqq c(\sigma)^{2} \int\left\|\left(H_{0}+I\right)^{2} \theta\left(k^{\prime \prime}\right)\right\|^{2} d k^{\prime \prime} \\
\leqq c(\sigma)^{2}\left\|\left(H_{0}+I\right)^{2} \varphi\right\|^{2}
\end{gathered}
$$


and $c(\sigma) \rightarrow 0$. Now the Wick product $: \Gamma Q_{2 \varrho}{ }^{m} R: \varphi$ is obtained by applying the $m$ annihilation operators in $\Gamma Q_{2 \rho}{ }^{m}$ to the $m$ meson variables $k^{\prime \prime}$ in $(R \theta)\left(k^{\prime \prime}\right)$. Our previous results do not apply since $R \theta$, as a function of $k^{\prime \prime}$ and its other meson variables, is not symmetric and so is not in $\mathbf{F}$. However the proofs are still correct and we can see that : $\Gamma Q_{2 \varrho} R: \varphi$ is a state in $\mathbf{F}$. This depends upon the fact that $R \theta$ is zero if one of the variables $k_{j}$ in $k^{\prime \prime}$ has a magnitude greater than $K$. It also depends on the $L_{2}$ estimates for (1.11), as in the proof of Theorem 2.2.1. Thus a') is proved.

To prove $b^{\prime}$ ) we observe that

$$
\begin{aligned}
\|: \Gamma Q_{2 \varrho \sigma}{ }^{m} R(\sigma) & : \varphi-: \Gamma Q_{2 \varrho} R: \varphi \| \leqq \\
& \leqq\left\|: \Gamma Q_{2 \varrho \sigma}{ }^{m}(R(\sigma)-R): \varphi\right\|+\left\|:\left(\Gamma Q_{2 \varrho \sigma}{ }^{m}-\Gamma Q_{2 \varrho}{ }^{m}\right) R: \varphi\right\| .
\end{aligned}
$$

The operators $\Gamma Q_{2 \varrho \sigma}{ }^{m}$ are bounded uniformly in $\sigma$ when restricted to states with a bounded number of particles and with a bound on the support of the meson variables. This depends upon the $L_{2}$ estimates for (1.11) and it remains true even when the annihilators in $\Gamma Q_{2}{ }^{m}$ are applied to $k^{\prime \prime}$. Thus the first term tends to zero by (2.2.2). In the same way it follows from (2.2.1) and the convergence of the kernels in $\Gamma Q_{2 \varrho \sigma}$ that the second term tends to zero.

Lemma 2.2.2. Let $0 \leqq \tau<2^{-1}$ and let $\lambda$ and $\varrho$ be given. Then

$$
T_{2 \varrho}\left(\mathscr{D}_{T} \cap \mathscr{D}_{H_{0}}\right) \subset \mathscr{F}(\lambda, \tau) \subset \mathscr{E}(\lambda) \text {. }
$$

Proof. Since $T_{2 \varrho} \mathscr{D}_{T}$ contains only states with a finite number of particles, the only nonobvious fact to be proved is that

$$
T_{2 \varrho}\left(\mathscr{D}_{T} \cap \mathscr{D}_{H_{0}}\right) \subset \mathscr{D}_{F^{\prime}(\tau)} \text {. }
$$

However if $\varphi \in \mathscr{D}_{T} \cap \mathscr{D}_{H_{0}}$ then $\varphi \in \mathscr{D}_{F(\tau)}$ also and

$$
F_{\tau} T_{2 \varrho} \varphi=F_{\tau} \exp \left(-\Gamma Q_{2 \varrho}\right) \varphi=:\left(R(2, \tau)_{\varrho}+F_{\tau}\right) \exp \left(-\Gamma Q_{2 \varrho}\right): \varphi
$$

is in $\mathbf{F}$ by Lemma 2.2.1 and Theorem 2.2.2.

Remark. The same proof shows that $T_{2 \varrho \sigma} \varphi \in \mathscr{F}(\lambda, \tau)$ and $F_{\tau}\left(T_{2 \varrho \sigma}-T_{2 \varrho}\right) \varphi \rightarrow 0$ for $\varphi \in \mathscr{D}_{T} \cap \mathscr{D}_{H_{0}}$.

\subsection{Properties of $T_{10}$}

Theorem 2.3.1. Let $\lambda \geqq 0$ and $\tau \in[0,1 / 2)$. For all sufficiently large $\lambda_{1}$ and $\varrho$, the power series for $T_{1 \varrho}$ and $T_{1 \varrho \sigma}$ converge to define $T_{1 \varrho}$ and $T_{1 \varrho \sigma}$ as invertible operators from $\mathscr{E}\left(\lambda_{1}\right)$ into $\mathscr{E}(\lambda)$ and from $\mathscr{F}\left(\lambda_{1}, \tau\right)$ into $\mathscr{F}(\lambda, \tau)$. When restricted to a subspace of $\mathbf{F}$ with a bounded number of particles, $T_{10}$ and $T_{1 \varrho \sigma}$ are bounded operators.

Theorem 2.3.2. Let $\varrho$ be sufficiently large. Then

$$
T_{1 \varrho}-T_{1 \varrho \sigma} \rightarrow 0
$$

uniformly on any subspace of $\mathbf{F}$ with a bounded number of particles. 
Theorem 2.3.3. Let $\varrho$ be sufficiently large. Then

is a dense subset of $\mathbf{F}$.

$$
\mathscr{D}_{H_{1}}=T_{\varrho}\left(\mathscr{D}_{T} \cap \mathscr{D}_{H 0}^{2}\right)
$$

Theorem 2.3.4. Let $0 \leqq \tau<1 / 2$. Then the domain of the operator $F(\tau)$ contains $\mathscr{D}_{H_{1}}$. Let $0 \leqq \tau<1$. Then $F(\tau)$ defines a bilinear form on $\mathscr{D}_{H_{1}} \times \mathscr{D}_{H_{1}}$. For $\varphi \in \mathscr{D}_{T} \cap \mathscr{D}_{H_{0}}$ we have

$$
F(\tau)^{1 / 2} T_{\varrho \sigma} \varphi \rightarrow F(\tau)^{1 / 2} T_{\varrho} \varphi .
$$

These four theorems are based upon the following lemma, whose proof is postponed to Sec. 2.4.

Lemma 2.3.1. Let $\tau \in[0,1 / 2)$. Then

$$
\begin{array}{ll}
R(1, \tau)_{\varrho}(N+I)^{-1}, & R(1, \tau)_{\varrho \sigma}(N+I)^{-1}, \\
R(3, \tau)_{\varrho}(N+I)^{-1}, & R(3, \tau)_{\varrho \sigma}(N+I)^{-1}
\end{array}
$$

are bounded operators from $\mathbf{F}$ into $\mathbf{F}$, and their norms tend to zero uniformly in $\sigma$ as $\varrho \rightarrow \infty$. As $\sigma \rightarrow \infty$

$$
\begin{aligned}
& \left\|\left(R(1, \tau)_{\varrho \sigma}-R(1, \tau)_{\varrho}\right)(N+I)^{-1}\right\| \rightarrow 0 \\
& \left\|\left(R(3, \tau)_{\varrho \sigma}-R(3, \tau)_{\varrho}\right)(N+I)^{-1}\right\| \rightarrow 0 .
\end{aligned}
$$

Proof of Theorem 2.3.1. We give the proof only for $T_{1 \varrho}$, since the proof for $T_{1 \varrho \sigma}$ is identical. If $\varphi \in \mathscr{E}\left(\lambda^{\prime}\right)$ then

and if $\psi=T_{1 \varrho} \varphi$,

$$
\left\|\varphi_{k}\right\| \leqq \text { const. } e^{-\lambda^{\prime} k / 2},
$$

$$
\begin{gathered}
\left\|\psi_{n}\right\| \leqq \sum_{(n / 4) \leqq j+k \leqq n}\left\|(j !)^{-1}\left(\Gamma Q_{1 \varrho}+\Gamma Q_{3 \varrho}\right)^{j} \varphi_{k}\right\| \leqq \\
\leqq \\
\sum_{(n / 4) \leqq j+k \leqq n}\left\|\left(\Gamma Q_{1 \varrho}+\Gamma Q_{3 \varrho}\right)(N+I)^{-1}\right\|^{j} 4^{j} \frac{(j+k) !}{j ! k !}\left\|\varphi_{k}\right\| \leqq \\
\sum_{(n / 4) \leqq j+k \leqq n}\left\|\left(\Gamma Q_{1 \varrho}+\Gamma Q_{3 \varrho}\right)(N+I)^{-1}\right\|^{j} 4^{j} 2^{n}\left\|\varphi_{k}\right\| .
\end{gathered}
$$

By Lemma 2.3.1 in the case $\tau=0$,

$$
4\left\|\left(\Gamma Q_{1 \varrho}+\Gamma Q_{3 \varrho}\right)(N+I)^{-1}\right\| \leqq e^{-\lambda^{\prime}}
$$

for large $\varrho$ (depending on $\lambda^{\prime}$ ). Thus

and

$$
\left\|\psi_{n}\right\|^{2} \leqq \text { const. } n^{4} 2^{2 n} e^{-\lambda^{\prime} n}
$$

$$
\sum_{n} e^{\lambda n}\left\|\psi_{n}\right\|^{2} \leqq \text { const. } \sum n^{4} 2^{2 n} e^{\left(\lambda-\lambda^{\prime}\right) n}
$$

which is finite for $\lambda^{\prime}>\lambda+2 \ln 2$. Thus $T_{1 \varrho} \varphi \in \mathscr{E}(\lambda)$.

If $\varphi$ has at most $n_{0}$ particles then

$$
\begin{gathered}
\left\|\psi_{n}\right\|^{2} \leqq \text { const. } n^{4} 2^{2 n} e^{-\lambda^{\prime} n} e^{\lambda^{\prime} n_{0}}\|\varphi\|^{2}, \\
\sum_{n}\left\|\psi_{n}\right\|^{2} \leqq \text { const. } e^{\lambda^{\prime} n_{0}}\|\varphi\|^{2}
\end{gathered}
$$

where the constants do not depend on $\varphi$. 
We choose

$$
\lambda_{1}>\lambda^{\prime}+2 \ln 2, \quad \lambda^{\prime}>\lambda+2 \ln 2 .
$$

Then $T_{1 \varrho}$ maps $\mathscr{E}\left(\lambda_{1}\right)$ into $\mathscr{E}\left(\lambda^{\prime}\right) \subset \mathscr{E}(\lambda)$ and by the same estimates as above, $\exp \left(\Gamma Q_{1 \varrho}+\Gamma Q_{3 \varrho}\right)$ converges absolutely as an operator from $\mathscr{E}\left(\lambda^{\prime}\right)$ into $\mathscr{E}(\lambda)$. Thus the power series for

$$
\exp \left(\Gamma Q_{1 \varrho}+\Gamma Q_{3 \varrho}\right) T_{1 \varrho}=\exp \left(\Gamma Q_{1 \varrho}+\Gamma Q_{3 \varrho}\right) \exp \left(-\Gamma Q_{1 \varrho}-\Gamma Q_{3 \varrho}\right)
$$

converges absolutely as an operator from $\mathscr{E}\left(\lambda_{1}\right)$ into $\mathscr{E}(\lambda)$. After rearrangement, this series converges to $I$, so $T_{1 \varrho}$ is invertible. This proves Theorem 2.3.1 as far as $\mathscr{E}(\lambda)$ is concerned.

Now suppose $\varphi \in \mathscr{F}\left(\lambda_{1}, \tau\right)$. Then

$$
F_{\tau} T_{1 \varrho} \varphi=T_{1 \varrho}\left(R(1, \tau)_{\varrho}+R(3, \tau)_{\varrho}+F_{\tau}\right) \varphi .
$$

It follows from Lemma 2.3.1 that $R(1, \tau)_{\varrho} \varphi$ and $R(3, \tau)_{\varrho} \varphi$ are in $\mathscr{E}\left(\lambda_{1}-\varepsilon\right)$ for any $\varepsilon>0$. Thus

$$
\begin{aligned}
& \left(R(1, \tau)_{\varrho}+R(3, \tau)_{\varrho}+F_{\tau}\right) \varphi \in \mathscr{E}\left(\lambda_{1}-\varepsilon\right) \\
& T_{1 \varrho}\left(R(1, \tau)_{\varrho}+R(3, \tau)_{\varrho}+F_{\tau}\right) \varphi \in \mathscr{E}(\lambda) .
\end{aligned}
$$

It follows that $T_{1 e} \varphi \in \mathscr{F}(\lambda, \tau)$ and the proof is complete.

Proof of Theorem 2.3.2. The operators $\Gamma Q_{i_{\varrho} \sigma}$, etc. which we are considering contain only creation operators. Thus they commute with one another and this enables us to use the formula

$$
a^{j}-b^{j}=(a-b) \sum_{i=0}^{j-1} a^{i} b^{j-i-1}
$$

from commutative algebra. Let $\varphi$ be a state in $\mathbf{F}$ with at most $n_{0}$ particles. For brevity we let

$$
\begin{aligned}
& a=\left(-\Gamma Q_{1 \varrho \sigma}-\Gamma Q_{3 \varrho \sigma}\right)(N+I)^{-1} \\
& b=\left(-\Gamma Q_{1 \varrho}-\Gamma Q_{3 \varrho}\right)(N+I)^{-1}
\end{aligned}
$$

If $\psi=\left(T_{1 \varrho \sigma}-T_{1 \varrho}\right) \varphi$, then

$$
\begin{aligned}
\left\|\psi_{n}\right\| & \leqq \sum_{(n / 4) \leqq j+k \leqq n}(j !)^{-1}\left\|\left[(a(N+I))^{j}-(b(N+I))^{j}\right] \varphi_{k}\right\| \leqq \\
& \leqq n_{0}\|\varphi\|_{(n / 4)-n_{0} \leqq j \leqq n}\left\|a^{j}-b^{j}\right\| 4^{j} \frac{\left(j+n_{0}\right) !}{j ! n_{0} !} \leqq \\
& \leqq \text { const. }\|\varphi\|_{(n / 4)-n_{0} \leqq j \leqq n} \sum^{j}\|a-b\| \sum_{i=0}^{j-1}\|a\|\|b\| j-i-1 \leqq \\
& \leqq \text { const. }\|\varphi\|\|a-b\| \sum_{(n / 4)-n_{0} \leqq j \leqq n}(8\|a\|+8\|b\|)^{j} \leqq \\
& \leqq \text { const. }\|\varphi\|\|a-b\|(8\|a\|+8\|b\|)^{(n / 4)-n_{0}}
\end{aligned}
$$

if $\varrho$ is sufficiently large, so that

$$
8\|a\|+8\|b\|<1 .
$$


If $n \leqq 4 n_{0}$ we have the more elementary estimate

Thus

$$
\begin{aligned}
\left\|\psi_{n}\right\| & \leqq \text { const. }\|\varphi\| \sum_{j \leqq 4}\left\|a^{j}-b^{j}\right\| \leqq \\
& \leqq \text { const. }\|\varphi\|\|a-b\| \quad(\|a\|+\|b\|+1)^{4 n_{0}} \leqq \\
& \leqq \text { const. }\|\varphi\|\|a-b\| .
\end{aligned}
$$

$$
\|\psi\|^{2} \leqq \text { const. }\|\varphi\|^{2}\|a-b\|^{2}
$$

and this tends to zero as $\sigma \rightarrow \infty$, in view of Lemma 2.3.1 and the definition of $a$ and $b$.

Proof of Theorem 2.3.3. From Theorems 2.2.1 and 2.3.1 we see that $T_{1 \varrho} \varphi$ is in the closure $\mathscr{D}_{H_{1}}$ of $\mathscr{D}_{H_{1}}$ if $\varphi$ is any state with a finite number of particles. Let $\theta$ be a state with a finite number of particles. It is sufficient to prove that $\theta \in \mathscr{D}_{\bar{H}_{1}}$. We choose a $\lambda$ such that

$$
\lambda>-2 \ln 8\|b\|+2 \ln 2
$$

( $b$ is from the proof of Theorem 2.3.2) and such that $T_{1 e}$ is defined as an operator on $\mathscr{E}(\lambda)$. We let

$$
\varphi=T_{1 \varrho}^{-1} \theta \in \mathscr{E}(\lambda)
$$

If $\varphi_{\leqq k}$ is the projection of $\varphi$ onto the subspace of $\mathbf{F}$ of states with at most $k$ particles, then it is sufficient to prove that

since $T_{1 \varrho} \varphi_{\leqq k} \in \mathscr{D}_{\bar{H}_{1}}$ and $T_{1 \varrho} \varphi=\theta$.

$$
T_{1 \varrho} \varphi \leqq k \rightarrow T_{1 \varrho} \varphi
$$

We have

$$
\begin{aligned}
\|\left(T_{1 \varrho}(\varphi \leqq k-\varphi)_{n} \|\right. & \leqq \sum_{\substack{(n / 4) \leqq j+l \\
l<<}}\|b\|^{j} 4^{j} \frac{(j+l) !}{j ! l !}\left\|\varphi_{l}\right\| \leqq \\
& \leqq \text { const. } \sum_{\substack{(n / 4) \\
k<l}} \sum_{\substack{k \leq j \leqq n \\
<l}}(8\|b\|)^{j} 2^{l} \exp (-\lambda l / 2)
\end{aligned}
$$

since $\varphi \in \mathscr{E}(\lambda)$. This is bounded by

const. $2^{k} \exp (-\lambda k / 2)(8\|b\|)^{(n / 4)-k} \leqq$

$$
\leqq \text { const. }(8\|b\|)^{n / 4} \exp (k(\ln 2-(\lambda / 2)-\ln 8\|b\|)) .
$$

We note that the coefficient of $k$ is negative and that

$$
\left\|T_{1 \varrho}(\varphi \leqq k-\varphi)\right\|^{2} \leqq \text { const. } \exp (2 k(\ln 2-(\lambda / 2)-\ln 8\|b\|)) .
$$

This tends to zero as $k \rightarrow \infty$, and the proof is complete.

Proof of Theorem 2.3.4. Let $\tau \in[0,1 / 2)$ and let $\varphi \in \mathscr{D}_{T} \cap \mathscr{D}_{H_{0}}$. We use formulas from the proof of Theorem 2.3.1 and Lemma 2.2.2 to obtain

$$
\begin{aligned}
F_{\tau} T_{\varrho} \varphi & =F_{\tau} T_{1 \varrho} T_{2 \varrho} \varphi \\
& =T_{1 \varrho}\left(R(1, \tau)_{\varrho}+R(3, \tau)_{\varrho}+F_{\tau}\right) T_{2 \varrho} \varphi \\
& =T_{1 \varrho}:\left(\sum_{i=1}^{3} R(i, \tau)_{\varrho}+F_{\tau}\right) T_{2 \varrho}: \varphi
\end{aligned}
$$

and a similar formula with the cutoff $\sigma$ present. By Lemmas 2.2.1 and 
2.3.1, the pair of operators

and

$$
F_{\tau}+\sum_{i=1}^{3} R(i, \tau)_{\varrho}
$$

$$
F_{\tau}+\sum_{i=1}^{3} R(i, \tau)_{\varrho \sigma}
$$

satisfy the hypotheses of Theorem 2.2.2. By the convergence in Theorems 2.2.2 and 2.3.2,

$$
F_{\tau} T_{\varrho \sigma} \varphi \rightarrow F_{\tau} T_{\varrho} \varphi
$$

This proves the first statement. The remaining statements follow from what we have proved together with the inequality

$$
F_{2 \tau}<F_{\tau}^{2} \text {. }
$$

\subsection{The basic Estimates}

The next theorem is an improvement on Lemma 1.1. It states that one of the fermions can be neglected in determining the bound on $R$ defined by (1.7). Let $N_{b}$ and $N_{f}$ be the number of bosons and number of fermions operators, respectively.

Theorem 2.4.1. Let $R$ be the operator defined by (1.7). Then

$$
\left\|\left(N_{f}+I\right)^{-\left(j_{1}-1\right) / 2}\left(N_{b}+I\right)^{-j_{2} / 2} R\right\| \leqq \text { const. }\|r\|_{2}
$$

provided $j_{1} \geqq 1$. The constant depends only on $j_{1}$ and $j_{2}$.

Proof. In the case $j_{1}=1, j_{2}=0$, this theorem is well known and the proof is based upon the fact that at most one fermion may be present in any given fermion state. We prove the theorem by induction. Suppose $j_{1} \geqq 2$ and suppose the theorem proved for operators $R$ depending on $j_{1}-1$ fermions and $j_{2}=0$ bosons. We make two simplifying assumptions. First we suppose that the fermion operator $b^{\#}\left(p_{1}\right)$ associated with the first variable in $r$ is a creator. (If this is not the case, replace $R$ by $R^{*}$ ). Second, we suppose that the kernel $r$ is a continuous function with compact support. (This assumption is permissible because it is sufficient to prove the theorem for a sequence $R_{n}$ of operators with kernels $r_{n}$ converging to $r$ in $L_{2}$.) Let

Then

$$
R\left(p_{1}\right)=\int r\left(p_{1}, p_{2}, \ldots\right) b^{\#}\left(p_{2}\right) \ldots b^{\#}\left(p_{j_{1}}\right) d p_{2} \ldots d p_{j_{1}} .
$$

and if $\varphi$ is a state then

$$
R=\int b^{*}\left(p_{1}\right) R\left(p_{1}\right) d p_{1}
$$

$$
\|R \varphi\|^{2} \leqq \int\left\|\left(N_{f}+I\right)^{1 / 2} R\left(p_{1}\right) \varphi\right\|^{2} d p_{1},
$$

by the definition of the creator $b^{*}$. By our induction hypothesis,

$$
\begin{aligned}
\left\|\left(N_{f}+I\right)^{-\left(j_{1}-1\right) / 2} R \varphi\right\|^{2} & \leqq \int\left\|\left(N_{f}+I\right)^{-\left(j_{1}-2\right) / 2} R\left(p_{1}\right) \varphi\right\|^{2} d p_{1} \leqq \\
& \leqq \text { const. }\|\varphi\|^{2} \int\left(\int\left|r\left(p_{1}, \ldots\right)\right|^{2} d p_{2} \ldots d p_{j_{1}}\right) d p_{1} \leqq \\
& \leqq \text { const. }\|\varphi\|^{2}\|r\|^{2} .
\end{aligned}
$$


This completes the induction and the theorem is proved whenever $i_{2}=0$. We use a similar induction on $j_{2}$ and thereby prove the theorem in general.

Remarks. Let $E$ be a measurable subset of the real numbers. Let $N_{f}(E)$ be the operator which measures the number of fermions with momenta in $E$. If $R$ creates or annihilates one fermion with momenta definitely in $E$ (that is, if there is a $j_{0}$ such that $r(k, p)=0$ whenever $\left.p_{j_{0}} \notin E\right)$ then one of the factors $\left(N_{f}+I\right)^{-1 / 2}$ in (2.4.1) may be replaced by the factor $\left(N_{f}(E)+I\right)^{-1 / 2}$. If another fermion in $R$ has momenta definitely in a set $F$ then another factor $\left(N_{f}+I\right)^{-1 / 2}$ may be replaced by $\left(N_{f}(F)+I\right)^{-1 / 2}$, etc. If $R$ has at least one fermion creator then $N_{f}+I$ in (2.4.1) may be replaced by $N_{f}$. If the creator has momentum definitely in $E$ then $\left(N_{f}(E)+I\right)^{-1 / 2}$ may be replaced by $N_{f}(E)^{-1 / 2}$. If $R$ has at least one fermion annihilator then $\left(N_{f}+I\right)^{-\left(j_{1}-1\right) / 2} R$ may be replaced by $R N^{-\left(j_{1}-1\right) / 2}$ in (2.4.1). The operator $N_{f}^{-\left(j_{1}-1\right) / 2}$ is only defined on the orthogonal complement of the zero fermion states. Similar considerations apply to the operator $N_{b}$ in (2.4.1). Next we describe a circumstance in which we can ignore two fermions. Suppose $r$ factors into a product, $r=s t$, where $s$ and $t$ depend on distinct variables and suppose $s$ and $t$ each depend on at least one fermion variable. Then

$$
\left\|\left(N_{f}+I\right)^{-\left(j_{1}-2\right) / 2}\left(N_{b}+I\right)^{-j_{2} / 2} R\right\| \leqq \text { const. }\|r\|_{2} .
$$

This inequality follows directly from (2.4.1).

The next result is known.

Theorem 2.4.2. Suppose the kernel $r$ of the operator $R$ is a smooth function with compact support. Then $\left(N_{b}+I\right)^{-j_{2} / 2} R$ is a bounded operator. The bound can be estimated by the diameter of the support of $r$ and by the $L_{2}$ norms of $r$ and a finite number of its derivatives.

Outline of proof. First suppose $j_{2}=0$. Choose a suitable orthonormal basis $\left\{e_{l}\right\}$ of $L_{2}$. (For example, and exponential $(2 \pi)^{-1 / 2} \exp (i n k)$ times the characteristic function of a periodic interval $2 \pi m \leqq k \leqq$ $\leqq 2 \pi(m+1)$ would be a suitable $\left.e_{l}\right)$. We expand $r$ as a sum of tensor products of basis elements,

The assumptions on $r$ imply that

$$
r=\Sigma \lambda_{l_{1}, \ldots, l_{j_{1}}} e_{l_{1}} \otimes \cdots \otimes e_{l_{j_{1}}} .
$$

$$
\Sigma\left|\lambda_{l_{1}, \ldots, l_{j_{1}}}\right|
$$

is finite, and this quantity provides a bound for $\|R\|$.

Next suppose $j_{2}>0$ and use induction on $j_{2}$ as in the proof of Theorem 2.4.1.

We say that an operator $B$ is infinitely small with respect to a positive operator $A$ if for each $\varepsilon>0$ there is a $K=K(\varepsilon)$ such that

$$
|(\psi, B \psi)| \leqq(\psi,(\varepsilon A+K I) \psi)
$$

for all $\psi$ in the domain of $A^{1 / 2}$. 
Corollary. Suppose $j_{1} \leqq 2$ and $j_{2} \leqq 1$ and suppose that $r \in L_{2}$. Then $R$ is infinitely small with respect to $N$. that

Proof. We can find a smooth function $r_{0}$ with compact support such

$$
\left\|r-r_{0}\right\|_{2} \leqq \varepsilon .
$$

Let $R_{0}$ be the operator with kernel $r_{0}$. Then

by Theorem 2.4.2 and

$$
\begin{aligned}
R_{0} & \leqq K_{1}\left(N_{b}+I\right)^{1 / 2} \leqq \\
& \leqq \varepsilon\left(N_{b}+I\right)+\varepsilon^{-1} K_{1}^{2} I
\end{aligned}
$$

$$
R-R_{0} \leqq \text { const. } \varepsilon(N+I)
$$

by Theorem 2.4.1. The constant depends only on $j_{1}$ and this completes the proof.

The operators we will deal with do not all have $L_{2}$ kernels and so the theorems we have proved will not be strong enough. The kernels fail to be in $L_{2}$ due to an insufficiently rapid decrease for large momenta. The operator $F_{\tau}$ is much larger than $N$ when the momenta are large, and so we can obtain better estimates if we dominate $R$ by powers of $F_{\tau}$ instead of dominating it by powers of $N$. Let $F_{\tau b}$ be the boson part of $F_{\tau}$.

Lemma 2.4.1. Let $R\left(k_{1}\right)$ be a bounded operator depending continuously in the norm topology on the variable $k_{1}$. Let $R($. ) have compact support and let

Then

$$
R=\int a^{*}\left(k_{1}\right) R\left(k_{1}\right) d k_{1}
$$

$$
\begin{gathered}
\left\|\left(N_{b}+I\right)^{-1 / 2} R\right\|^{2} \leqq \int\left\|R\left(k_{1}\right)\right\|^{2} d k_{1} \\
\left\|\left(F_{\tau b}+I\right)^{-1 / 2} R\right\|^{2} \leqq \int \mu\left(k_{1}\right)^{-\tau}\left\|R\left(k_{1}\right)\right\|^{2} d k_{1} \\
\left\|\left(F_{\tau b}+I\right)^{-1 / 4}\left(N_{b}+I\right)^{-1 / 4} R\right\|^{2} \leqq \int \mu\left(k_{1}\right)^{-\tau / 2}\left\|R\left(k_{1}\right)\right\|^{2} d k_{1} .
\end{gathered}
$$

Proof. The first inequality is elementary and was used in the proof of Theorem 2.4.1. We omit the proof. To prove the second inequality, let

$$
\begin{aligned}
R(A) & =\int_{A} a^{*}(k) R(k) d k \\
F_{\tau b}(A) & =\int_{A} \mu(k) a^{*}(k) a(k) d k .
\end{aligned}
$$

Let $\left\{A_{1}, A_{2}, \ldots\right\}$ be a partition of the real numbers into disjoint measurable sets. Then

$$
\begin{aligned}
|(\psi, R \varphi)| & =\sum_{j}\left|\left(\psi, R\left(A_{j}\right) \varphi\right)\right| \leqq \\
& \leqq \sum_{j}\left\|\left(F_{\tau b}\left(A_{j}\right)+I\right)^{1 / 2} \psi\right\|\left\|\left(F_{\tau b}\left(A_{j}\right)+I\right)^{-1 / 2} R\left(A_{j}\right)\right\|\|\varphi\| \leqq \\
& \leqq\left(\sum_{j}\left\|\left(F_{\tau b}\left(A_{j}\right)+I\right)^{1 / 2} \psi\right\|^{2} \sum_{j}\left\|\left(F_{\tau b}\left(A_{j}\right)+I\right)^{-1 / 2} R\left(A_{j}\right)\right\|\right)^{1 / 2}\|\varphi\| \\
& =\left\|\left(F_{\tau b}+I\right)^{1 / 2} \psi\right\|\left(\sum_{j}\left\|\left(F_{\tau b}\left(A_{j}\right)+I\right)^{-1 / 2} R\left(A_{j}\right)\right\|^{2}\right)^{1 / 2}\|\varphi\| .
\end{aligned}
$$


If we substitute $\left(F_{\tau b}+I\right)^{-1 / 2} \psi$ for $\psi$ we can conclude that

Let

$$
\left\|\left(F_{\tau b}+I\right)^{-1 / 2} R\right\|^{2} \leqq \sum_{j}\left\|\left(F_{\tau b}\left(A_{j}\right)+I\right)^{-1 / 2} R\left(A_{j}\right)\right\|^{2} .
$$

Then

$$
\mu_{* j}=\inf _{k \in A_{j}} \mu(k) .
$$

$$
\left\|\left(F_{\tau b}+I\right)^{-1 / 2} R\right\|^{2} \leqq \sum_{j} \mu_{* j}{ }^{-\tau}\left\|\left(N_{b}\left(A_{j}\right)+I\right)^{-1 / 2} R\left(A_{j}\right)\right\|^{2} .
$$

At this point we use a refined form of the first inequality :

Thus

$$
\left\|\left(N_{b}\left(A_{j}\right)+I\right)^{-1 / 2} R\left(A_{j}\right)\right\|^{2} \leqq \int_{A_{j}}\|R(k)\|^{2} d k .
$$

$$
\left\|\left(F_{\tau b}+I\right)^{-1 / 2} R\right\|^{2} \leqq \sum_{j} \mu_{* j}{ }^{-\tau} \int_{A_{j}}\|R(k)\|^{2} d k .
$$

As the mesh length goes to zero, we obtain

$$
\left\|\left(F_{\tau b}+I\right)^{-1 / 2} R\right\|^{2} \leqq \int \mu^{-\tau}\|R(k)\|^{2} d k .
$$

To prove the third inequality we note that

$$
\sum_{i=1}^{n} \mu\left(k_{i}\right)^{\tau / 2} \leqq n^{1 / 2}\left(\sum_{i=1}^{n} \mu\left(k_{i}\right)^{\tau}\right)^{1 / 2} .
$$

Thus $F_{(\tau / 2) b} \leqq F_{\tau b}{ }^{1 / 2} N^{1 / 2}$ and the left hand side of (2.4.5) is bounded by $\left\|\left(F_{(\tau / 2) b}+I\right)^{-1 / 2} R\right\|^{2}$ and so (2.4.5) follows from (2.4.4).

By passing to adjoints we can estimate $\int R(k) a(k) d k$. We can obtain similar bounds for fermion operators $\int b^{*}(p) R(p) d p$ and their adjoints. If $R($.$) has support contained in a set E$ then $\left(N_{b}+I\right)^{-1 / 2}$ or $\left(F_{\tau b}+I\right)^{-1 / 2}$ can be replaced by $\left(N_{b}(E)+I\right)^{-1 / 2}$ or by $\left(F_{\tau b}(E)+I\right)^{-1 / 2}$.

We return to the operator $R$ of Theorem 2.4.1. We divide the variables of $r$ into three disjoint sets, $A, B$, and $C$. Let the variables $k_{j}$ and $p_{i}$ in $C$ be associated only with creators, let the variables $k_{j}$ and $p_{i}$ in $A$ be associated only with annihilation operators, and let the variables in $B$ be either creation or annihilation variables. Let $|A|$ be the number of variables in $A$, etc. Let $E_{A}=\omega\left(p_{i}\right) \mu(k) \ldots$ be the product of the energies of the variables in $A$. Let $E_{C}$ be defined similarly.

Theorem 2.4.3. Suppose $B$ contains at least one fermion variable. Then $\left\|\left(F_{\tau}+I\right)^{-|C| / 2} R\left(F_{\tau}+I\right)^{-|A| / 2}(N+I)^{-(|B|-1) / 2}\right\|$

and

$$
\leqq \text { const. }\left\|E_{A}^{-\tau / 2} E_{C^{-\tau / 2}} r\right\|_{2}
$$

$$
\begin{array}{r}
\left\|\left(F_{\tau}+I\right)^{-|C| / 4} R\left(F_{\tau}+I\right)^{-|A| / 4}(N+I)^{-((|B|-1) / 2)-(|A|+|C|) / 4}\right\| \\
\leqq \text { const. }\left\|E_{A}^{-\tau / 4} E_{C}^{-\tau / 4} r\right\|_{2} .
\end{array}
$$

The constants depend only on the number of variables in $r$.

Proof. We proceed by induction on $|A|+|C|$. If $|A|+|C|=0$ we are in the case of Theorem 2.4.1. Suppose $|A|+|C|>0$ and suppose for 
example that $|C|>0$ and that one of the variables in $C$ is $k_{1}$, a meson variable. The operator on the left side of (2.4.6) can be written

where

$$
\left(F_{\tau}+I\right)^{-1 / 2} \int a^{*}\left(k_{1}\right) P\left(k_{1}\right) d k_{1}
$$

$$
R=\int a^{*} R\left(k_{1}\right) d k_{1}
$$

as in the proof of Theorem 2.4.1 and

$$
P\left(k_{1}\right)=\left(F_{\tau}+I+\mu\left(k_{1}\right)\right)^{-(|C|-1) / 2} R\left(k_{1}\right)\left(F_{\tau}+I\right)^{-|A| / 2}(N+I)^{-(|B|-1) / 2} .
$$

Now

$$
\left\|\left(F_{\tau}+I\right)^{1 / 2}\left(F_{\tau}+I+\mu\left(k_{1}\right)\right)^{-1 / 2}\right\|=1,
$$

so our induction hypothesis implies that

$$
\left\|P\left(k_{1}\right)\right\|^{2} \leqq \text { const. } \int \mu^{\tau}\left(k_{1}\right) E_{A}^{-\tau} E_{C}^{-\tau}\left|r\left(k_{1}, \ldots\right)\right|^{2} d k_{2} \ldots
$$

Assume that the kernel $r$ of $R$ is continuous with compact support. Then the hypothesis of Lemma 2.4.1 is satisfied, and so

$$
\left\|\left(F_{\tau}+I\right)^{-1 / 2} \int a^{*}\left(k_{1}\right) P\left(k_{1}\right) d k_{1}\right\|^{2} \leqq \text { const. } \int E_{A}{ }^{-\tau} E_{C}{ }^{-\tau}|r|^{2} d k_{1} d k_{2} \ldots
$$

The case of a general kernel follows from the case of a continuous kernel with compact support by limits. This proves (2.4.6). The proof of $(2.4 .7)$ is similar.

Remarks. There are some obvious refinements in this theorem. We may replace some of the factors $\left(F_{\tau}+I\right)^{-1 / 2}$ by boson operators $\left(F_{\tau b}+I\right)^{-1 / 2}$ and we may replace some by fermion operators $\left(F_{\tau f}+I\right)^{-1 / 2}$.If certain particles are created or annihilated only with momenta in some set $E$ then we can replace the corresponding operators $\left(F_{\tau}+I\right)^{-1 / 2}$ by the operators $\left(F_{\tau}(E)+I\right)^{-1 / 2}$. If the kernel $r$ factors into a product, $r=s t$, and if $s$ and $t$ depend upon distinct variables and if each factor contributes a fermion to $B$ then one of the factors $\left(N_{f}+I\right)^{-1 / 2}$ in $(2.4 .6)$ and (2.4.7) may be omitted. This does not follow from the theorem but is proved by induction, as the theorem was.

To illustrate what this theorem means, we list the three special cases which arise from a trilinear boson fermion coupling.

Case 1. $R=\int r a^{*}(k) b^{*}\left(p_{1}\right) b^{* *}\left(p_{2}\right) d k d p_{1} d p_{2}$

Case 2. $R=\int r a(k) b^{*}\left(p_{1}\right) b^{*}\left(p_{2}\right) d k d p_{1} d p_{2}$

Case 3. $R=\int r a^{*}(k) b^{*}\left(p_{1}\right) b\left(p_{2}\right) d k d p_{1} d p_{2}$

Corollary. Suppose

Case 1. $\mu^{-\tau / 2} r \in L_{2}$ or $\omega_{1}^{-\tau / 2} r \in L_{2}$ or $\omega_{2}^{-\tau / 2} r \in L_{2}$

Case 2. $\mu^{-\tau / 2} \omega_{1}^{-\tau / 2} r \in L_{2}$ or $\mu^{-\tau / 2} \omega_{2}^{-\tau / 2} r \in L_{2}$

Case 3. $\mu^{-\tau / 2} \omega_{2}^{-\tau / 2} r \in L_{2}$.

Then $R$ is infinitely small with respect to $F_{\tau}$.

Remark. The restrictions on $r$ in case 1 are the strongest, and the renormalization associated with this term is the most likely to be infinite. 
Proof. We consider only case 3 . We find a smooth function $r_{0}$ with compact support such that

$$
\left\|\mu^{-\tau / 2} \omega_{2}^{-\tau / 2}\left(r-r_{0}\right)\right\|_{2}<\varepsilon .
$$

If $R_{0}$ is the operator with kernel $r_{0}$ then $R_{0}$ is infinitely small with respect to $F_{\tau}$ by the corollary to Theorem 2.4.2. Also

Thus

$$
\left.\| F_{\tau}+I\right)^{-1 / 2}\left(R-R_{0}\right)\left(F_{\tau}+I\right)^{-1 / 2} \| \leqq \text { const } \varepsilon .
$$

$$
\begin{aligned}
\left|\left(\psi,\left(R-R_{0}\right) \psi\right)\right| & \leqq \text { const. } \varepsilon\left\|\left(F_{\tau}+I\right)^{1 / 2} \psi\right\|^{2} \leqq \\
& \leqq \text { const. } \varepsilon\left(\psi,\left(F_{\tau}+I\right) \psi\right) .
\end{aligned}
$$

This completes the proof in case 3 . The remaining cases are similar.

Proof of Lemma 2.3.1. The assertions concerning $R(1, \tau)$ follow from Theorem 2.4.1 and Lemma 1.2. The treatment of $R(3, \tau)$ will also be based on Theorem 2.4.1. Because we need to be able to neglect the effect of two fermions in $R(3, \tau)$ instead of the one allowed by Theorem 2.4.1, the reduction is now more complicated. Let

$$
r=\left(\Sigma \omega_{i}^{\tau}\right)\left(\Sigma \omega_{i}\right)^{-1}\left(\omega_{3}+\omega_{4}+\mu\right)^{-1} \tilde{q}_{2}\left(p_{1}, p_{2}, k\right) \tilde{q}_{1}\left(p_{3}, p_{4}, k\right) .
$$

Then

$$
\int\left(\int|r|^{2} d p\right)^{1 / 2} d k \leqq\left\|\left(\omega_{1}+\omega_{2}\right)^{-(1-\tau)} \tilde{q}_{2}\right\|_{2}\left\|\left(\omega_{3}+\omega_{4}+\mu\right)^{-1} \tilde{q}_{1}\right\|_{2}
$$

and the norms on the right are finite. (See Lemma 1.2 and its proof.) Thus for almost every $k$,

$$
R(k)=\int r(p, k) b^{*}\left(p_{1}\right) b^{* *}\left(p_{2}\right) b^{*}\left(p_{3}\right) b^{* *}\left(p_{3}\right) d p
$$

defines an operator on $\mathbf{F}$ (Lemma 1.1) and

$$
\begin{gathered}
R(3, \tau)=\int R(k) d k \\
\left\|R(3, \tau)(N+I)^{-1}\right\| \leqq \int\left\|R(k)(N+I)^{-1}\right\| d k .
\end{gathered}
$$

There is a similar inequality between the cutoff operators $R(3, \tau)_{e}$ and $R(3, \tau)_{\varrho \sigma}$ and the corresponding $k$ dependent operators $R(k)_{e}$ and $R(k)_{\rho \sigma}$.

Because of the factor $\left(\sum_{i} \omega_{i}^{\tau}\right)$, the kernel $r(., k)$ is a sum of four terms. Except for the factor $\left(\sum_{i} \omega_{i}\right)^{-1}$, each term splits into two factors, the first depending only on $p_{1}$ and $p_{2}$ and the second depending only on $p_{3}$ and $p_{4}$. Let $f_{j}$ be the characteristic function of the interval $\left[j \omega_{0},(j+1) \omega_{0}\right)$. Let

Then

$$
\begin{aligned}
& \delta_{j} \omega=\omega_{3}+\omega_{4}-j \omega_{0} \\
& \omega^{(j)}=\omega_{1}+\omega_{2}+j \omega_{0} .
\end{aligned}
$$

$$
\begin{aligned}
\left(\Sigma \omega_{i}\right)^{-1} & =\sum_{j=1}^{\infty} f_{j}\left(\omega_{3}+\omega_{4}\right)\left(\omega^{(j)}+\delta_{j} \omega\right)^{-1} \\
& =\sum_{j m} f_{j}\left(\omega_{3}+\omega_{4}\right)\left(\omega^{(j)}\right)^{-m-1}\left(-\delta_{j} \omega\right)^{m} .
\end{aligned}
$$


We substitute this in one of the four terms contributing to $r(., k)$ and we obtain infinite series $\sum_{j m} r_{j m}(., k)$. Furthermore each term in this series factors,

$$
r_{j m}(p, k)=s_{j m}\left(p_{1}, p_{2}, k\right) t_{j m}\left(p_{3}, p_{4}, k\right) .
$$

Where $f_{j}\left(\omega_{3}+\omega_{4}\right) \neq 0$ we have $\left|\delta_{j} \omega\left(\omega^{(j)}\right)^{-1}\right| \leqq 2^{-1}$ and so

$$
\sum_{j m}\left(\int\left|r_{j m}\right|^{2} d p\right)^{1 / 2} \leqq 2 \sum_{j}\left(\int\left|r_{j 0}\right|^{2} d p\right)^{1 / 2} .
$$

We assert that

$$
\int \sum_{j}\left(\int\left|r_{j 0}(p, k)\right|^{2} d p\right)^{1 / 2} d k
$$

is finite. By Theorem 2.4.1, we then conclude that

$$
\int\left\|R(k)(N+I)^{-1}\right\| d k
$$

is finite. We have a similar inequality for $R(k)_{\varrho}$ and $R(k)_{\varrho \sigma}$ and this tells us the operators

$$
R(3, \tau)_{\varrho}(N+I)^{-1}, \quad R(3, \tau)_{\varrho \sigma}(N+I)^{-1}
$$

of Lemma 2.3.1 are bounded. The difference between these operators and their limits (as $\varrho \rightarrow \infty$ or $\sigma \rightarrow \infty$ ) can be estimated by the same argument. For example

$$
\left\|R(3, \tau)_{\varrho \sigma}(N+I)^{-1}\right\|+\left\|R(3, \tau)_{\varrho}(N+I)^{-1}\right\|
$$

is bounded by a finite sum of terms like

$$
\int \sum_{j m}\left(\int_{\left|p_{i}\right|>\varrho}\left|r_{j m}(p, k)\right|^{2} d p\right)^{1 / 2} d k .
$$

This term tends to zero uniformly in $\sigma$ by the Lebesgue dominated convergence theorem. Thus the lemma will follow from this assertion.

The integral we asserted to be finite is bounded by

$$
\begin{gathered}
\sum_{j=1}^{\infty} \int\left(\int\left(\omega^{(j)}\right)^{-2}\left(\sum \omega_{i}^{\tau}\right)^{2}\left(\omega_{3}+\omega_{4}+\mu\right)^{-2}\left|\tilde{q}_{2}\right|^{2} f_{j}\left|\tilde{q}_{1}\right|^{2} d p\right)^{1 / 2} d k \leqq \\
\leqq\left(\int\left(\omega_{1}+\omega_{2}\right)^{-1-\gamma}\left|\tilde{q}_{2}\right|^{2} d p_{1} d p_{2} d k\right)^{1 / 2} \times \\
\times \sum_{j=1}^{\infty}\left(\int\left(j \omega_{0}\right)^{-1-\gamma}\left(\omega_{3}+\omega_{4}+\mu\right)^{-1-\gamma} f_{j}\left|\tilde{q}_{1}\right|^{2} d p_{3} d p_{4} d k\right)^{1 / 2}
\end{gathered}
$$

for some positive $\gamma$ depending on $\tau$. The first integral is finite. (See the proof of Lemma 1.2.) Thus the above quantity is bounded by

const. $\left(\sum_{j=1}^{\infty} j^{-1-\gamma}\right)^{1 / 2}\left(\sum_{j=1}^{\infty}\left\|\left(\omega_{3}+\omega_{4}+\mu\right)^{-(1-\gamma) / 2} f_{j} \tilde{q}_{1}\right\|^{2}\right)^{1 / 2} \leqq$

and this is finite by Lemma 1.2 .

$$
\leqq \text { const. }\left\|\left(\omega_{3}+\omega_{4}+\mu\right)^{-(1-\gamma) / 2} \tilde{q}_{1}\right\|_{2}
$$




\section{The Definition of $\boldsymbol{H}_{\text {ren }}$ (Beginning)}

\subsection{Introduction}

In Sec. 3 and Sec. 4 we define $H_{\text {ren }}$. In Sec. 3 we show that $H_{1}\left(=H_{\text {ren }}-V_{2}\right)$ is an operator on the dense domain $\mathscr{D}_{H_{1}}$ constructed in Sec. 2. This means that if $\psi \in \mathscr{D}_{H_{1}}$ then $H_{1} \psi$ can be defined and is in $\mathbf{F}$. The definition of $H_{1}$ is the main step in defining $H_{\text {ren }}$. The reason for this is that $H_{1}$ contains all of the infinite renormalizations in this model. (The fermion mass renormalization, which is associated with $V_{2}$, is finite, and we omit it entirely.) In addition to defining $H_{1} \psi$ we show that our definition agrees with a limit of cutoff renormalized operators $H_{1 \sigma}$. For $\varphi \in \mathscr{D}_{T}$ we set

$$
\psi_{\sigma}=T_{Q \sigma} \varphi
$$

and we recall that in Sec. 2 we showed

$$
\lim _{\sigma \rightarrow \infty} \psi_{\sigma}=\psi
$$

exists (and equals $T_{e} \varphi$ ). We will show

$$
\lim _{\sigma \rightarrow \infty} H_{1 \sigma} \psi_{\sigma}=H_{1} \psi
$$

Thus the quadratic forms converge,

$$
\left(\psi_{\sigma}, H_{1 \sigma} \psi_{\sigma}\right) \rightarrow\left(\psi, H_{1} \psi\right) .
$$

It will follow from this that $H_{1}$ is a symmetric operator.

In Sec. 3.2 we choose the finite $\sigma$-dependent renormalization terms $L(\sigma)$ for the cutoff operator $H_{1 \sigma}$. Then

We will see that

$$
H_{1 \sigma}=H_{0}+V_{1 \sigma}+L(\sigma) \text {. }
$$

$$
H_{0} T_{\varrho \sigma}=T_{\varrho \sigma} H_{0}-T_{1 \varrho \sigma}: Q_{\varrho \sigma} T_{2 \varrho \sigma}:+T_{1 \varrho \sigma}:\left(Q_{2 \varrho \sigma}-\left[H_{0}, \Gamma Q_{2 \varrho \sigma}\right]\right) T_{2 \varrho \sigma}:
$$

We depend upon a formula of Friedrichs [2] which gives $P(\sigma)$ and $\widetilde{P}(\sigma)$ for which

$$
\begin{aligned}
V_{1 \sigma} T_{\varrho \sigma} & =T_{1 \varrho \sigma}: P(\sigma) T_{2 \varrho \sigma}: \\
L(\sigma) T_{\varrho \sigma} & =T_{1 \varrho \sigma}: \widetilde{P}(\sigma) T_{2 \varrho \sigma}: .
\end{aligned}
$$

This formula will be given in Sec. 3.3. To define $H_{1}$ as the limit of the $H_{1 \sigma}$ we add these formulas and obtain

$$
H_{1} T_{\varrho \sigma}=T_{\varrho \sigma} H_{0}+T_{1 \varrho \sigma}:\left(-Q_{\varrho \sigma}+P(\sigma)+\widetilde{P}(\sigma)+Q_{2 \varrho \sigma}-\left[H_{0}, \Gamma Q_{2 \varrho \sigma}\right]\right) T_{2 \varrho \sigma}:
$$

The last two terms in the parenthesis have a sum which approaches a limit (Lemma 2.2.1). It only remains to show

$$
-Q_{\varrho \sigma}+P(\sigma)+\widetilde{P}(\sigma)
$$


approaches a limit as $\sigma \rightarrow \infty$. None of the three terms in (3.1.1) approaches a limit as $\sigma \rightarrow \infty$ and so we must take advantage of cancellations to find a limit for the sum. These cancellations, of course, are the well known infinite cancellations of Quantum Field Theory (in so far as they are present in the model we consider). These cancellations are performed in Sec. 3.5 and Sec. 3.6. There are two reasons why the limit $P(\infty)$ of the $P(\sigma)$ 's does not exist. One portion of $P(\infty)$ maps vectors in $\mathbf{F}$ into functions which are not in $L_{2}$, due to the fact that they decrease too slowly at infinity. This part of $P(\infty)$ is cancelled by $-Q_{\varrho}$. Another part of $P(\infty)$ can be described loosely as in integral operator with a kernel identically equal to plus infinity. This part of $P(\infty)$ is cancelled by $\widetilde{P}(\infty)$.

\subsection{Definition of $H_{1 \sigma}$}

We now give the definition of a cutoff version, $H_{1 \sigma}$, of our operator $H_{1}$. This can be done directly because the renormalizations in $H_{1 \sigma}$ are finite. We set

$$
H_{1 \sigma}=H_{0}+V_{1 \sigma}+\delta m_{\sigma}^{2} \int: \Phi_{\sigma}(x)^{2}: h(x)^{2} d x+c_{\sigma} I .
$$

Here $\delta m_{\sigma}$ and $c_{\sigma}$ are the positive constants defined by the equations

$$
\begin{gathered}
\delta m_{\sigma}^{2}=4 \ln \left(2 \sigma_{f}+1\right) \\
c_{\sigma} I=V_{1 \sigma}-\bigcirc-Q_{1 \sigma} .
\end{gathered}
$$

The meaning of the symbol on the right side of (3.2.3) will be explained in Sec. 3.3 and does not concern us now.

Theorem 3.2.1. $H_{1 \sigma}$ is a symmetric operator on the dense domain $\mathscr{D}_{H_{1 \sigma}}$ defined by

and

$$
\mathscr{D}_{H_{1 \sigma}}=T_{\varrho \sigma}\left(\mathscr{D}_{T} \cap \mathscr{D}_{H_{0}}\right)
$$

$$
\begin{gathered}
H_{0} T_{\varrho \sigma} \varphi=T_{\varrho \sigma} H_{0}+T_{1 \varrho \sigma}\left(-Q_{\varrho \sigma}+Q_{2 \varrho \sigma}-\left[H_{0}, \Gamma Q_{2 \varrho \sigma}\right]\right) T_{2 \varrho \sigma} \varphi \\
\text { for } \varphi \in \mathscr{D}_{T} \cap \mathscr{D}_{H_{0}} .
\end{gathered}
$$

Proof. The results of Sec. 2 imply absolute convergence of the power series and so we may compute term by term. We get

$$
\begin{aligned}
{\left[H_{0}, T_{\varrho \sigma}\right] \varphi } & =\left[H_{0}, T_{1 \varrho \sigma}\right] T_{2 \varrho \sigma} \varphi+T_{1 \varrho \sigma}\left[H_{0}, T_{2 \varrho \sigma}\right] \varphi \\
& =-T_{1 \varrho \sigma}\left[H_{0}, \Gamma Q_{\varrho \sigma}\right] T_{2 \varrho \sigma} \varphi
\end{aligned}
$$

and this gives us (3.2.4). The operator $V_{1 \sigma}$ has an $L_{2}$ krenel, so $V_{1 \sigma}$ is an operator on the domain of $N^{3 / 2}$, and $V_{1 \sigma}$ is defined on $\mathscr{D}_{H_{1}}$. We compute the kernel of

Let

$$
\int: \Phi_{\sigma}(x)^{2}: h(x)^{2} d x
$$

$$
\Phi_{\sigma}(k)=a_{\sigma}(k)+a_{\sigma}^{*}(-k) .
$$


Then (3.2.5) equals

$$
\begin{aligned}
& \int h(x)^{2}: \Phi_{\sigma}(k) \Phi_{\sigma}(l): e^{i(k+l) x}(\mu(k) \mu(l))^{-1 / 2} d x d k d l \\
= & \int(\hat{h} * \hat{h})(-k-l): \Phi_{\sigma}(k) \Phi_{\sigma}(l):(\mu(k) \mu(l))^{-1 / 2} d k d l .
\end{aligned}
$$

The kernel is in $L_{\mathbf{2}}$. (This is independent of the momentum cutoff $\sigma$ on the field $\Phi_{\sigma}$.) Thus (3.2.5) is an operator defined on $\mathscr{D}_{N}$ and on $\mathscr{D}_{H_{1}}$ for $\sigma \leqq \infty$.

\subsection{Diagrams and Attached Products}

We use diagrams in the sense of FRIEDRICHS [2] to express operators. A diagram with $l$ solid legs pointing to the left and $m$ pointing to the right denotes an operator which annihilates $m$ nucleons or antinucleons and creates $l$ of them. Similarly the dotted lines denote boson annihilators and boson creators. The diagram then represents an operator such as (1.7), that is the integral of a function times the Wick ordered product of the annihilation and creation operators indicated by the diagram. The function is the numerical kernel of the operator and we see that the operator is uniquely determined by its numerical kernel together with
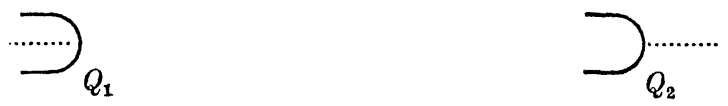

Fig. 1

its diagram. For example $Q_{1}$ and $Q_{2}$ have diagrams given by Fig. 1. The product $R S$ of two such operators $R$ and $S$ does not have the same form since the annihilation operators in $R$ follow the creation operators in $S$. However by use of commutation (or anticommutation) relations we can replace $a(k) a^{*}\left(k^{\prime}\right)$ by $a^{*}\left(k^{\prime}\right) a(k)+\delta\left(k-k^{\prime}\right)$ (for example). After a number of such replacements, the product $R S$ is expressed as a sum of terms of the form (1.7). In each term a certain number $c_{b}$ of the pairs $a(k) a^{*}\left(k^{\prime}\right)$ have been replaced by a $\delta\left(k-k^{\prime}\right)$ and a certain number $c_{f}$ of the fermion pairs $b\left(p_{1}\right) b^{*}\left(p_{2}\right)$ or $b^{\prime}\left(p_{1}\right) b^{*}\left(p_{2}\right)$ have been replaced by $\delta\left(p_{1}-p_{2}\right)$. We denote this term by the symbol

$$
R-0-S
$$

and call it the contribution to the product with $c_{f}$ fermion contractions and $c_{b}$ boson contractions. We associate a diagram to this operator. It is the diagram obtained by joining $c_{f}$ of the annihilating fermion legs

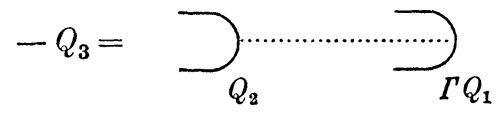

Fig. 2

of $R$ each with a creating fermion leg of $S$, and similarly connecting $c_{b}$ of the boson legs. That is, the legs corresponding to contracted pairs are joined. Thus $-Q_{3}$ is given by Fig. 2 . 
Following Friedrichs [2] we define a connected product

$$
P<: R_{1} \ldots R_{n}:
$$

to be the sum of all contributions to the ordinary product

$$
P: R_{1} \ldots R_{n} \text { : }
$$

in which at least one creating leg from each factor $R_{j}$ is contracted with an annihilating leg from $P$. If $n=0$, we define the connected product to be $P$. In particular we will be concerned with

$$
V_{\sigma}<: \Gamma Q_{Q}{ }^{n}: .
$$

Since $V_{\sigma}$ has only three legs, this connected product is zero if $n \geqq 4$. Of basic importance to us is Friedrichs' formula [2]

$$
V_{1} T_{\varrho}=\sum_{n=0}^{\infty}(-1)^{n}(n !)^{-1}:\left(V_{1}<: \Gamma Q_{\varrho}{ }^{n}:\right) T_{\varrho}:
$$

In our case this reduces to

$V_{1} T_{e}=T_{1 \varrho}:\left(V_{1}-V_{1}-O-\Gamma Q_{\varrho}+2^{-1} V_{1} \angle: \Gamma Q_{\varrho}{ }^{2}:-6^{-1} V_{1}<: \Gamma Q_{\varrho}{ }^{3}:\right) T_{2 \varrho}:$

The quantity in the parenthesis is the limit $P(\infty)$ of the term $P(\sigma)$ of (3.1.1). Let $\Delta_{\sigma}$ be the cutoff boson mass renormalization counterterm proportional to (3.2.5). Friedrichs' formula also tells us an expression for

$\left(\Delta_{\sigma}+c_{\sigma} I\right) T_{\varrho \sigma}=T_{1 \varrho \sigma}:\left(c_{\sigma} I+\Delta_{\sigma}-\Delta_{\sigma}-O-\Gamma Q_{\varrho \sigma}+2^{-1} \Delta_{\sigma} \angle: \Gamma Q_{\varrho \sigma}{ }^{2}:\right) T_{2 \varrho \sigma}:$.

The quantity in the parentheses is the term $\widetilde{P}(\sigma)$ of (3.1.1).

Since the right sides of these formulas (3.3.1) and (3.3.2) appear considerably more complicated than the left sides, we explain why the formulas are useful. The formulas express an ordinary product (of an operator times a Wick exponential) as a sum of terms, each one of which is Wick ordered. Thus each term has the form of the operator $R$ of (1.7). Such an operator causes trouble only when the kernel $r$ is bad. Thus the infinities appear in the kernels of certain terms in the parentheses in (3.3.1) and (3.3.2). It is easy to identify the infinite terms and to pair them together in such a way that the infinities cancel. For example the key cancellation of the theory involves the boson mass renormalization and occurs in the sum

$$
\Delta_{\text {ren }}(\sigma)=\Delta_{\sigma}-V_{1 \varrho \sigma}-0-\left(\Gamma Q_{1 \varrho \sigma}+\Gamma Q_{2 \varrho \sigma}\right) .
$$

In Sec. 3.5 we show that $\Delta_{\text {ren }}(\sigma)$ has a finite limit as $\sigma \rightarrow \infty$.

We now give Friedrichs' formal derivation of his formula. Any product $V: G^{n}$ : can be written as a sum of those contributions in which $V$ is contracted with $j$ of the factors of: $G^{n}$ :, letting $j$ run from 0 to $n$. 
The contribution with $j$ factors contracted is

$$
\left(\begin{array}{l}
n \\
j
\end{array}\right):\left(V \leftarrow: G^{j}:\right) G^{n-j}:
$$

since there are $n ! / j !(n-j) !$ choices of the $j$ factors. Thus

and

$$
V: G^{n}:=\sum_{j}\left(\begin{array}{l}
n \\
j
\end{array}\right):\left(V<: G^{j}:\right) G^{n-j}:
$$

$$
\begin{aligned}
V: e^{G}: & =\Sigma(n !)^{-1} V: G^{n}: \\
& =\Sigma((n-j) !)^{-1}(j !)^{-1}:\left(V \perp: G^{j}:\right) G^{n-j}: \\
& =\Sigma(j !)^{-1}:\left(V \perp: G^{j}:\right)\left(: e^{G}:\right): \\
& =:\left(V \perp: e^{G}:\right)\left(: e^{G}:\right): .
\end{aligned}
$$

\subsection{Convergent Contributions to $H_{1}$}

The formula (3.3.1) is formal and it contains divergent integrals, i.e. infinities. The next lemma deals with those contributions to (3.3.1) which are not divergent.

Lemma 3.4.1. Let $R(\sigma)$ be any one of the following operators and let $R$ be the corresponding operator obtained by omitting the cutoff $\sigma$. Then hypotheses a) and b) of Theorem 2.2.2 are satisfied.

$$
\begin{array}{rl} 
& -6^{-1} V_{1 \sigma} \perp: \Gamma Q_{\varrho \sigma}{ }^{3}: \\
2^{-1} V_{1 \sigma} & <:\left(\Gamma Q_{1 \varrho \sigma}+\Gamma Q_{2 \varrho \sigma}\right)^{2}:-A_{\sigma} \\
2^{-1} V_{1 \sigma} & <: \Gamma Q_{3 \varrho \sigma}{ }^{2}: \\
V_{1 \sigma} & <:\left(\Gamma Q_{1 \varrho \sigma}+\Gamma Q_{2 \varrho \sigma}\right) \Gamma Q_{3 \varrho \sigma}:-B_{\sigma} \\
& -V_{1 \sigma}-0-\Gamma Q_{3 \varrho \sigma}+C_{\sigma} \\
& -V_{1 \sigma}-0-\Gamma Q_{\varrho \sigma}-V_{1 \sigma}-0-\Gamma Q_{\varrho \sigma} . \\
1,0 & 1,1
\end{array}
$$

Definition of $A$ and $A_{\sigma}, B$ and $B_{\sigma}, C$ and $C_{\sigma}$. The quantities $A, B$ and $C$ are infinite quantities given by certain terms which contribute to $2^{-1} V_{1}<:\left(\Gamma Q_{1 \varrho}+\Gamma Q_{2 \varrho}\right)^{2}$ :, etc. The difference

$$
2^{-1} V_{1}\left\llcorner: \Gamma Q_{\varrho}^{2}:-A\right.
$$

(for example) is defined as the sum of all terms contributing to the first part $2^{-1} V_{1}<: \Gamma Q_{\mathrm{e}}{ }^{2}$ : which do not contribute to the second part, $A$. The terms contributing to $A, B$ and $C$ are expressed by the diagrams in Fig. 3. $A_{\sigma}, B_{\sigma}$ and $C_{\sigma}$ are obtained by replacing $V_{1}$ by $V_{1 \sigma}$ and $Q_{\varrho}$ by $Q_{\varrho \sigma}$ in these diagrams, the integrals involved in defining $A_{\sigma}$ etc. are finite and $A_{\sigma}$, etc. are operators (Lemma 1.1). A could equivalently be defined by the formula

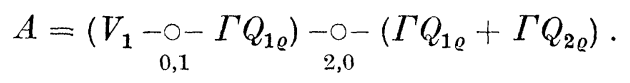



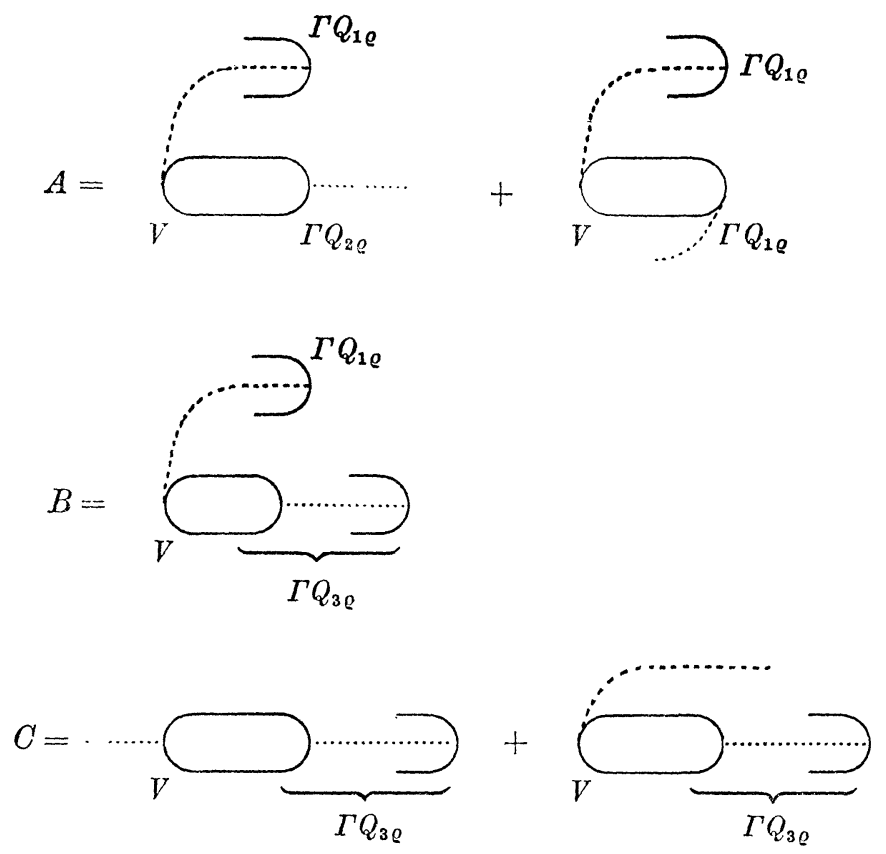

Fig. 3

This has only a formal interpretation, but the corresponding formula

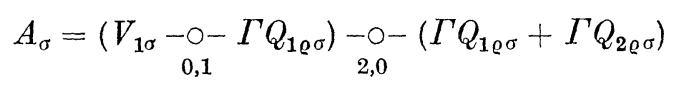

has a rigorous meaning. To get a similar description of $B$ and $C$, we must work with the definition (1.6) of the kernel of $Q_{3}$. Both $B$ and $C$ consist of all terms in which both fremion legs from $Q_{2 \varrho}$ (as part of $\Gamma Q_{3 \varrho}$ ) are contracted with $V_{1}$. In other words, in the $B$ and $C$ terms, the variables $p_{1}$ and $p_{2}$ of $\Gamma Q_{3}$ are contracted with $V_{1}$. Now $B_{\sigma}$ has a simple definition in terms of $C_{\sigma}$,

$$
B_{\sigma}=\underset{0,1}{C-O-} \Gamma Q_{1 \varrho \sigma}
$$

but $C_{\sigma}$ cannot be expressed as an attached product of $V$ and the $\Gamma Q_{i}$. Thus we write out the integral defining $C_{\sigma}$.

$$
\begin{aligned}
C_{\sigma}=- & \int b^{*}\left(p_{1}\right) b^{\prime *}\left(p_{2}\right)\left[a^{*}(k) \tilde{q}_{2 \sigma}^{-}\left(p^{\prime}, p^{\prime \prime}, k\right)+a(k) \tilde{q}_{1 \sigma}^{-}\left(p^{\prime}, p^{\prime \prime}, k\right)\right] \times \\
& \times\left(\omega^{\prime}+\omega^{\prime \prime}+\omega_{1}+\omega_{2}\right)^{-1}\left(\omega_{1}+\omega_{2}+\mu(l)\right)^{-1} \times \\
& \times \tilde{q}_{2 \varrho \sigma}\left(p^{\prime}, p^{\prime \prime}, l\right) \tilde{q}_{1 \varrho \sigma}\left(p_{1}, p_{2}, l\right) d p d k d l .
\end{aligned}
$$

Proof of Lemma 3.4.1. Only the fermion pair annihilation terms from $V_{1}$ enter in these attached products. Except for five terms (certain 
contributions to (3.4.2) and (3.4.4) and all of (3.4.6)) both fermion legs from $V_{1}$ are contracted and they are contracted either with distinct factors of : $\Gamma Q_{Q}{ }^{2}$ : or of : $\Gamma Q_{Q}{ }^{3}$ : or else with two variables of $\Gamma Q_{3 e}$ and in this case the variables are not both from $Q_{20}$. We multiply and divide by $\omega^{\tau}, 1 / 4<\tau<1 / 2$, in each of the integrals corresponding to the fermion contractions in (3.4.1)-(3.4.5). We multiply the $\Gamma Q_{\varrho}$ factor by $\omega\left(p_{j}\right)^{\tau}$ and divide the $V$ factor by $\omega\left(p_{j}\right)^{\tau}$, where $p_{j}$ is the variable in question. The result does not change the products $(3.4 .1)-(3.4 .5)$ but $V_{1}$ is replaced by an operator $W$ with a kernel $w$ bounded by

$$
\text { const. } \mu(k)^{-(1 / 2)+\varepsilon} \omega\left(p_{1}\right)^{-\tau} \omega\left(p_{2}\right)^{-\tau}\left(\left| \pm k+p_{1}+p_{2}\right|+1\right)^{-(1 / 2)+\varepsilon},
$$

$\varepsilon=(\tau-(1 / 4)) / 2>0$. Along rays through the origin this function is bounded by const. $(\varrho+1)^{-(3 / 2)-\varepsilon}$. Here $\varrho$ is the distance from the origin and the constant depends upon the ray. However the constant is in $L_{2}$ on spheres, so $w$ is in $L_{2}$. The factor from $\Gamma Q_{\varrho}$ is also replaced by a new operator and the new operator has a domain containing $\mathscr{D}_{T}$. To see this we consider several cases. $\Gamma Q_{1 e}$ is replaced by an operator whose kernel is bounded by the magnitude of the kernel of $R(1, \tau)$, and this was shown to be in $L_{2}$. When restricted to a fixed subspace $D(n, K)$, $\Gamma Q_{2 \varrho}$ is replaced by an operator with an $L_{2}$ kernel. (See formula (1.11) of Lemma 1.2.) $\Gamma Q_{3 e}$ is replaced by an operator whose kernel is bounded by the $L_{2}$ function (1.10). Thus the new kernels are all in $L_{2}$ and the assertions a) and b) now follow from Lemma 1.1.

The exceptional terms can be treated by a similar argument. Here we make use of the fact that $\varphi \in \mathscr{D}_{H_{0}}$. Thus if $\varphi$ is multiplied by $\omega\left(p_{j}\right)$, the resulting state has a finite norm. $\left(\omega\left(p_{j}\right) \varphi\right.$ is in $L_{2}$, but is not antisymmetric in the $p$ 's.) This completes the proof.

The fermion pair creation part of $V_{1}$ is $Q_{1}+Q_{2}$, and the fermion pair annihilation part of $V_{1}$ is $V_{1}-Q_{1}-Q_{2}$. The latter also gives a convergent contribution to (3.3.1).

Lemma 3.4.2. Let

$$
\begin{aligned}
R(\sigma) & =V_{1}-Q_{1 \varrho \sigma}-\left[H_{0}, \Gamma Q_{2 \varrho \sigma}\right] \\
R & =V_{1}-Q_{1 \varrho}-\left[H_{0}, \Gamma Q_{2 \varrho}\right] .
\end{aligned}
$$

Then hypotheses a) and $b$ ) of Theorem 2.2.2. are satisfied.

Proof. By Lemma 2.2.1 we can replace the commutators $\left[H_{0}, \Gamma Q_{2 \varrho \sigma}\right]$ and $\left[H_{0}, \Gamma Q_{2 \varrho}\right]$ by $Q_{2 \varrho \sigma}$ and $Q_{2 \varrho}$ respectively. The operators $Q_{1}-Q_{1 \varrho}$ and $Q_{2}-Q_{2 \varrho}$ have integrands which vanish for large fermion momenta, and it is not hard to see that the integrands are in $L_{2}$. In view of Lemma 1.1 it is sufficient to consider

$$
\begin{aligned}
R^{\prime}(\sigma) & =V_{1 \sigma}-Q_{1 \sigma}-Q_{2 \sigma} \\
R^{\prime} & =V_{1}-Q_{1}-Q_{2} .
\end{aligned}
$$


The fermion operators in $R^{\prime}$ and $R^{\prime}(\sigma)$ are annihilators. Thus we can multiply and divide by $\omega\left(p_{i}\right), i=1,2$ in the products $R^{\prime} \varphi$ and $R^{\prime}(\sigma) \varphi$, where $\varphi \in D(n, K) \cap \mathscr{D}_{H_{0}^{2}}$. This is equivalent to replacing $R^{\prime}$ by an operator with a kernel in $L_{2}$. (See formula (1.12) from the proof of Lemma 1.2.) Also the state $\varphi$ is replaced by an unsymmetrized state $\tilde{\varphi}$ and

$$
\|\tilde{\varphi}\| \leqq\left\|\left(H_{0}+I\right)^{2} \varphi\right\|<\infty .
$$

Thus $R\left(H_{0}+I\right)^{-2}$ is a bounded operator from $D(n, K) \cap \mathscr{D}_{H_{0}^{2}}$ into $\mathbf{F}$, and the same estimates show that $R^{\prime}(\sigma)-R^{\prime}$ tends to zero as $\sigma \rightarrow \infty$, as required by $b$ ).

The same argument proves

Lemma 3.4.3. Let

$$
\begin{gathered}
R(\sigma)=-V_{1 \sigma}-\circ-\Gamma Q_{1 \varrho \sigma}-Q_{3 \varrho \sigma} \\
R=-V_{1,1}^{-O-} \Gamma Q_{1 \varrho}-Q_{3 \varrho} .
\end{gathered}
$$

Then hypotheses $a$ ) and $b$ ) of Theorem 2.2.2 are satisfied.

\subsection{The Divergent Boson Self Energy Contribution to $H_{1}$}

Let $D_{\varrho \sigma}$ be the operator

$$
D_{\varrho \sigma}=V_{1 \sigma}-0-\left(\Gamma Q_{1 \varrho \sigma}+\Gamma Q_{2 \varrho \sigma}\right)
$$

and let $\Delta_{\sigma}$ be the cutoff boson mass renormalization counterterm in (3.2.1). If the cutoff $\sigma$ is removed from either of these operators, an infinite expression is obtained which does not define an operator. Nonetheless their difference

$$
\Delta_{\mathrm{ren}}=\Delta-D_{Q}
$$

can be defined as a limit of cutoff differences

$$
\Delta_{\mathrm{ren}}(\sigma)=\Delta_{\sigma}-D_{\varrho \sigma} .
$$

Lemma 3.5.1. Let $R(\sigma)$ denote one of the operators

$$
\begin{aligned}
& \Delta_{\text {ren }}(\sigma) \\
& \Delta_{\text {ren }}(\sigma) \underset{0,1}{-\circ-} \Gamma Q_{1 \varrho \sigma} \\
& \Delta_{\text {ren }}(\sigma) \underset{0,2}{-O-:} \Gamma Q_{1 \varrho \sigma}{ }^{2}:
\end{aligned}
$$

Then the limit $R$ exists and hypotheses a) and b) of Theorem 2.2.2 are satisfied. In (3.5.2) and (3.5.3) the limit can be evaluated by removing the cutoff,

$$
\begin{gathered}
\lim _{\sigma} \Delta_{\text {ren }}(\sigma)-0-\Gamma Q_{1 \varrho \sigma}=\Delta_{\text {ren }}-0-\Gamma Q_{1 \varrho} \\
0,1 \\
\lim _{\sigma} \Delta_{\text {ren }}(\sigma) \underset{0-0}{0,2} \Gamma Q_{1 \varrho \sigma}{ }^{2}:=\Delta_{\text {ren }} \underset{0,2}{-0-:} \Gamma Q_{1 \varrho}{ }^{2}:,
\end{gathered}
$$

with strong limits on $D_{T}$. 
Proof. $\Delta_{\text {ren }}(\sigma)$ divides naturally into a sum of four terms and each of the terms has the form of (1.7) with an $L_{2}$ kernel. These kernels, of course, depend on $\sigma$, and as $\sigma \rightarrow \infty$ we will prove that they converge in $L_{2}$ to limits. All the statements of the lemma follow directly from this convergence, with the help of the Lemma 1.1. The limits of the kernels permit us to define the operator $\Delta_{\text {ren }}$. The limits are the kernels of four operators whose sum is $\Delta_{\text {ren }}$ :

The proof that the kernels converge is complicated by the fact that we consider several different regions of integration and that we use different estimates to obtain convergence in each region. If $\Omega$ is a measurable set in Euclidean four space $E^{4}$, we define

$$
\begin{gathered}
\Delta_{\sigma}(\Omega)= \\
\underset{\substack{(p, k, l) \in \Omega \\
|\xi| \leqq 2 \sigma_{f}}}{ } \hat{h}(\eta-l) \hat{h}(-\eta-k): \Phi_{\varrho}(k) \Phi_{\sigma}(l): \\
(1+|\xi|)^{-1}(\mu(k) \mu(l))^{-1 / 2} d p d k d l .
\end{gathered}
$$

We have $\Delta_{\sigma}\left(E^{4}\right)=\Delta_{\sigma}$ because

and

$$
2^{-1} d \eta d \xi=d p_{1} d p_{2}=d p
$$

We also define

$$
\delta m_{\sigma}=4 \ln \left(2 \sigma_{f}+1\right)=2 \int_{-2 \sigma}^{2 \sigma_{f}}(1+|\xi|)^{-1} d \xi .
$$

$$
\begin{gathered}
D_{\varrho \sigma}(\Omega)=\int_{\Omega} \chi_{\varrho \sigma} \hat{h}(\eta-l) \hat{h}(-\eta-k): \Phi_{\sigma}(k) \times \\
\times\left(\frac{a_{\sigma}(l)}{\omega_{1}+\omega_{2}-\mu-i}+\frac{a_{\sigma}^{*}(-l)}{\omega_{1}+\omega_{2}+\mu}\right):(\mu(k) \mu(l))^{-1 / 2} S\left(p_{1}, p_{2}\right)^{2} d p d k d l
\end{gathered}
$$

where $\chi_{\varrho \sigma}$ is the fermion cutoff function, $\mu=\mu(l)$ and $S$ is given by (1.1) and (1.2). Here also we have $D_{\varrho \sigma}\left(E^{4}\right)=D_{\varrho \sigma}$.

The expansion of $\Delta_{\sigma}$ as a sum of four terms comes from substituting the definitions

$$
\Phi_{\sigma}(k)=a_{\sigma}(k)+a_{\sigma}^{*}(-k) ; \quad \Phi_{\sigma}(l)=a_{\sigma}(l)+a_{\sigma}^{*}(-l)
$$

in our formula for $\Delta_{\sigma}$. In the same way we have $D_{\varrho \sigma}$ and $\Delta_{\text {ren }}(\sigma)$ written as a sum of four terms. When $\varrho=0$ in $D_{\varrho \sigma}(\Omega)$, the lower or $\varrho$ cutoff is missing entirely. We call this operator $D_{\sigma}(\Omega)$.

We introduce the region

$$
\Sigma=\left\{\left(p_{1}, p_{2}, k, l\right):|\xi| \geqq 2|\eta|\right\} .
$$

We will prove that $D_{\sigma}(\sim \Sigma)$ and $\Delta_{\sigma}(\sim \Sigma)$ each have limits as $\sigma \rightarrow \infty$. Thus the infinite mass renormalization is associated with the region $\Sigma$. We also introduce the region $\Lambda_{a}$ defined by the equation

$$
\left|\omega_{1}+\omega_{2}-\mu-i\right| \leqq \mu^{a} \text {. }
$$


We prove the convergence of the kernels in a sequence of lemmas. These lemmas will thus prove Lemma 3.5.1.

Lemma 3.5.2. The kernels of $D_{\sigma}\left(\Lambda_{3 / 8}\right)$ converge to a limit in $L_{2}$ as $\sigma \rightarrow \infty$.

Proof. The second and fourth quadrants of the $p_{1}, p_{2}$ plane contribute to the kernels of $D_{\sigma}\left(\Lambda_{3 / 8}\right)$ functions bounded by the function

const. $(|k+l|+1)^{-1} \mu(k)^{-1 / 2} \mu(l)^{-1 / 8} \int(|\eta-l|+1)^{-2} d \eta$,

which is in $L_{2}$ and does not depend on $\sigma$. In the first and third quadrants of the $p_{1}, p_{2}$ plane we use the bound

$$
S^{2} \leqq \text { const. }\left(\omega_{1}^{-1}+\omega_{2}^{-1}\right)^{2} .
$$

These two quadrants contribute to the kernels of $D_{\sigma}\left(\Lambda_{3 / 8}\right)$ functions bounded by the function

$$
\text { const. }(|k+l|+1)^{-1} \mu(k)^{-1 / 2} \mu(l)^{-1 / 8} \int_{\omega_{0}}^{2 \mu} \omega^{-1} d \omega,
$$

which is in $L_{2}$ and does not depend on $\sigma$. For fixed $l$, the definition of $\Lambda_{3 / 8}$ puts a bound on the magnitude of the $p_{i}$ which enter into $D_{\sigma}\left(\Lambda_{3 / 8}\right)$. Thus for fixed $l$, the kernels are independent of $\sigma$ if $\sigma$ is large. The lemma now follows from the Lebesgue bounded convergence theorem

Lemma 3.5.3. The kernels of $D_{\sigma}\left(\Lambda_{3 / 4}\right)$ converge to a limit in $L_{2}$ as $\sigma \rightarrow \infty$.

Proof. We can work in the complement to $\Lambda_{3 / 8}$ and thus we can use the estimate

$$
\left|\omega_{1}+\omega_{2}-\mu-i\right|^{-1} \leqq \mu^{-3 / 8} .
$$

The second and fourth quadrants of the $p_{1}, p_{2}$ plane contribute to the kernels of $D_{\sigma}\left(\Lambda_{3 / 4}\right)$ functions bounded by (3.5.4) and the first and third quadrants contribute functions bounded by (3.5.6). The kernels converge pointwise (for fixed $k$ and $l$ ) as before and the lemma follows from the bounded convergence theorem.

Lemma 3.5.4. The kernels of $D_{\sigma}(\sim \Sigma)$ converge to a limit in $L_{2}$ as $\sigma \rightarrow \infty$.

Proof. We work in $\sim \Lambda_{3 / 4}$ and thus we use the estimate

$$
\left|\omega_{1}+\omega_{2}-\mu-i\right|^{-1} \leqq \mu^{-3 / 4} \leqq \text { const. }\left(\omega_{1}+\omega_{2}\right)^{-3 / 4} .
$$

We introduce the cone $\Sigma^{\prime}$ defined by the inequality $|\eta| \geqq 2|\xi|$. The kernels of $D_{\sigma}\left(\Sigma^{\prime} \sim \Lambda_{3 / 4}\right)$ are bounded by

$$
\text { const. }(|k+l|+1)^{-1}\left(\mu(k) \mu(l)^{-1 / 2} \int\left(\omega_{1}+\omega_{2}\right)^{-2-(3 / 4)} d p .\right.
$$

(We have used (3.5.5).) On the complement of $\Sigma^{\prime}$,

$$
\left(\omega_{1}+\omega_{2}\right)^{-3 / 4} \leqq \text { const. }(|\xi|+1)^{-3 / 4} .
$$

Thus the kernels of $D_{\sigma}\left(\sim\left(\Sigma \cup \Sigma^{\prime} \cup \Lambda_{3 / 4}\right)\right)$ are bounded by

$$
\text { const. }(|k+l|+1)^{-1} \mu(k)^{-1 / 2} \mu(l)^{-1 / 8} M
$$


where

$$
\begin{aligned}
& M=\int \mu(l)^{-3 / 8}(|-\eta+l|+1)^{-2} \int_{-2|\eta|}^{2|\eta|}(|\xi|+1)^{-3 / 4} d \xi d \eta \leqq \\
& \leqq \text { const. } \int \mu(l)^{-3 / 8}|\eta|^{1 / 4}(|-\eta+l|+1)^{-2} d \eta
\end{aligned}
$$

is bounded independently of $l$. The kernels converge pointwise and the lemma is proved.

It is easy to prove that the kernels of $D_{\varrho \sigma}-D_{\sigma}$ converge.

Lemma 3.5.5. The kernels of $\Delta_{\sigma}(\sim \Sigma)$ converge to a limit in $L_{2}$ as $\sigma \rightarrow \infty$.

Proof. The kernels are bounded by a function of the form (3.5.7) where

$$
M=\int \mu(l)^{-3 / 8}(|-\eta+l|+1)^{-2} \int_{-2|\eta|}^{2|\eta|}(|\xi|+1)^{-1} d \xi d \eta
$$

is bounded independently of $l$. The kernels converge pointwise and the lemma is proved.

Lemma 3.5.6. The kernels of $\Delta_{\sigma}\left(\Sigma \cap \Lambda_{3 / 4}\right)$ converge in $L_{2}$ as $\sigma \rightarrow \infty$.

Proof. The kernels are bounded by a function of the form (3.5.7) where

$$
M=\int \mu(l)^{-3 / 8}(|-\eta+l|+1)^{-2} \int_{-2 \mu}^{2 \mu}(|\xi|+1)^{-1} d \xi d \eta .
$$

The cutoffs in $\Delta_{\sigma}$ and in $D_{\sigma}$ are defined differently. Before comparing these two operators, we eliminate this difference. Let $\Omega_{\sigma}$ be the region

$$
\left\{\left|p_{1}\right| \geqq \sigma_{f} \text { or }\left|p_{2}\right| \geqq \sigma_{f}\right\} \cap \Sigma \text {. }
$$

Lemma 3.5.7. The kernels of $\Delta_{\sigma}\left(\Omega_{\sigma}\right)$ converge in $L_{2}$ as $\sigma \rightarrow \infty$.

Proof. The kernels are bounded by (3.5.7) where

$$
\begin{aligned}
M= & \int_{|\eta| \leqq \sigma_{f}}|-\eta+l|^{-2} \int_{2 \sigma_{f}-|\eta|}^{2 \sigma_{f}}(|\xi|+1)^{-1} d \xi d \eta \leqq \\
& \leqq \text { const. } \ln \left(\frac{2 \sigma_{f}}{2 \sigma_{f}-\sigma_{f}}\right)=\text { const. }
\end{aligned}
$$

since $|\eta| \leqq 2^{-1}|\xi|$ in $\Omega_{\sigma}$. For fixed $\eta$, the integrals

$$
\int_{2 \sigma_{f}-|\eta|}^{2 \sigma_{f}}(|\xi|+1)^{-1} d \xi
$$

converge to zero and this implies that the kernels converge pointwise.

Lemma 3.5.8. The kernels of

converge in $L_{2}$ as $\sigma \rightarrow \infty$.

$$
\Delta_{\sigma}\left(\Sigma \sim\left(\Lambda_{3 / 4} \cup \Omega_{\sigma}\right)\right)-D_{\sigma}\left(\Sigma \sim \Lambda_{3 / 4}\right)
$$

Proof. In $\Sigma \sim \Lambda_{3 / 4}$ we have

$$
\left|4-S^{2}\right| \leqq \text { const. }(|\xi|+1)^{-2}
$$

$$
\left|(|\xi|+1)^{-1}-\left(\omega_{1}+\omega_{2}+\mu\right)\right|^{-1} \leqq \text { const. } \mu^{1 / 4}(|\xi|+1)^{-5 / 4}
$$

$\left|(|\xi|+1)^{-1}-\left(\omega_{1}+\omega_{2}-\mu-i\right)\right|^{-1} \leqq$ const. $\mu(|\xi|+1)^{-1}\left(\omega_{1}+\omega_{2}-\mu\right)^{-1}$

$$
\leqq \text { const. } \mu^{3 / 8}(|\xi|+1)^{-5 / 4} \text {. }
$$


Thus the kernels are bounded by (3.5.7) where

$$
M=\int(|-\eta+l|+1)^{-2} \int(|\xi|+1)^{-5 / 4} d \xi d \eta
$$

This completes the proof of Lemma 3.5.8 and Lemma 3.5.1.

\subsection{The Self Energy as a Divergent Internal Line in Other Diagrams}

We show that the infinities cancel in the sums

$$
\begin{aligned}
& -\Delta-0-\Gamma Q_{1 \varrho}-C+A=E \\
& 2^{-1} \Delta-0-\Gamma Q_{1 \varrho}{ }^{2}+B=F .
\end{aligned}
$$

To this end we define the sums

$$
\begin{gathered}
E_{\sigma}=-\Delta_{\sigma}-0-\Gamma Q_{1 \varrho \sigma}-C_{\sigma}+A_{\sigma} \\
F_{\sigma}=2^{-1} \Delta_{\sigma}^{-0-} \Gamma Q_{1 \varrho \sigma}{ }^{2}+B_{\sigma} .
\end{gathered}
$$

These sums are well defined, but the expressions (3.6.1) and (3.6.2) for $E$ and $F$ have only a formal meaning.

Lemma 3.6.1. There are operators $E$ and $F$ with the following property. The pair $E, E_{\sigma}$ and the pair $F, F_{\sigma}$ each satisfy the hypotheses of Theorem 2.2.2.

Proof. In view of Lemma 3.5.1 we can replace $\Delta$ by $D$ in the above formulas without loss of generality. Thus we define

$$
E_{\sigma}^{\prime}=-D_{\sigma, 1}^{-0-} \Gamma Q_{1 \varrho \sigma}-C_{\sigma}+A_{\sigma}
$$

and we find an $E^{\prime}$ so that $E_{\sigma}^{\prime}$ and $E^{\prime}$ satisfy the hypotheses of Theorem 2.2. Now $D_{\sigma}-0-\Gamma Q_{1 \varrho \sigma}$ can be written as the sum of two terms, one for each 0,1

of the two boson variables in $D_{\sigma}$ which can contract. In fact

$$
\begin{aligned}
D_{\sigma-\bigcirc-1} \Gamma Q_{1 \varrho \sigma}= & \left(V_{1 \sigma}-\bigcirc-\Gamma Q_{1 \varrho \sigma}\right)-\circ-\left(\Gamma Q_{1 \varrho \sigma}+\Gamma Q_{2 \varrho \sigma}\right)+ \\
& +\int b^{*}\left(p_{1}\right) b^{\prime *}\left(p_{2}\right)\left[a^{*}(k) \tilde{q}_{2 \sigma}^{-}\left(p^{\prime}, p^{\prime \prime}, k\right)+a(k) \tilde{q}_{1 \sigma}^{-}\left(p^{\prime}, p^{\prime \prime}, k\right)\right] \times \\
& \times\left(\omega^{\prime}+\omega^{\prime \prime}-\mu(l)-i\right)^{-1}\left(\omega_{1}+\omega_{2}+\mu(l)\right)^{-1} q_{2 \varrho \sigma}\left(p^{\prime}, p^{\prime \prime}, l\right) \times \\
& \times q_{1 \varrho \sigma}\left(p_{1}, p_{2}, l\right) d p d k d l .
\end{aligned}
$$

The first term is $A_{\sigma}$ itself (cf. (3.4.8)) and results from the contraction of the boson from the $V_{1 \sigma}$ part of $D_{\sigma}=V_{1 \sigma-0-}\left(\Gamma Q_{1 \varrho \sigma}+\Gamma Q_{2 \varrho \sigma}\right)$. We call the second term $C_{\sigma}^{\prime}$. It results from a contraction of the boson from the $\Gamma Q_{2 \varrho \sigma}$ part of $D_{\sigma}$. (See Fig. 4.)

We observe that the integrands of $-C_{\sigma}$ and $C_{\sigma}^{\prime}$ are almost identical (cf. 3.4.10) and differ only in the energies $\omega^{\prime}$, etc. which enter into the denominators. The kernel of the operator $-C_{\sigma}^{\prime}-C_{\sigma}$ is a function of the 


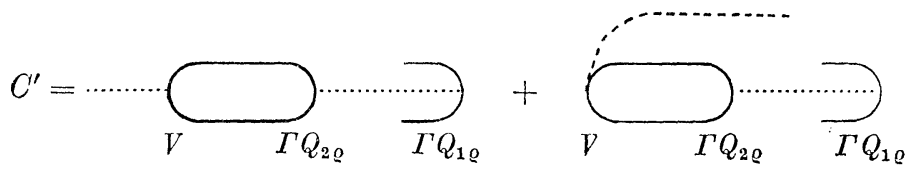

Fig. 4

variables $p_{1}, p_{2}, k$. We assert that the kernel is in $L_{2}$ as a function of these variables and converges in $L_{2}$ to a limit as $\sigma \rightarrow \infty$. This limit is then the kernel of an operator

$$
E^{\prime}=-C^{\prime}-C
$$

and the assertion will prove half of Lemma 3.6.1. However it will prove the other half also because one can check that

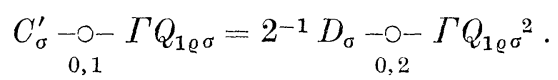

By definition of $B_{\sigma}$, we have

and hence

$$
\underset{0,1}{C_{\sigma}-\supset-\Gamma Q_{1 \rho \sigma}}=B_{\sigma}
$$

$$
\begin{aligned}
\lim _{\sigma} F_{\sigma} & =\lim _{\sigma}\left(2^{-1} \Delta_{\mathrm{ren}}(\sigma)-0-\Gamma Q_{1 \varrho \sigma^{2}}+2^{-1} D_{\sigma}^{-0-} \Gamma Q_{1 \varrho \sigma^{2}}+B_{\sigma}\right) \\
& =\lim _{\sigma}\left(2^{-1} \Delta_{\mathrm{ren}}(\sigma)-0-\Gamma Q_{1 \varrho \sigma^{2}}+\left(C_{\sigma}^{\prime}+C_{\sigma}\right)-0-\Gamma Q_{1 \varrho \sigma^{2}}\right) .
\end{aligned}
$$

Thus by Lemma 3.5.1 and our assertion, $F=\lim _{\sigma} F_{\sigma}$ exists and the pair $F, F_{\sigma}$ satisfy the hypotheses of Theorem 2.2.2.

To prove the assertion we deal separately with the contributions to $C_{\sigma}^{\prime}$ and $C_{\sigma}$ coming from the region $\Lambda_{3 / 4}$ described by the inequality

$$
\left|\omega^{\prime}+\omega^{\prime \prime}-\mu(l)\right| \leqq \mu(l)^{3 / 4} .
$$

By Lemma 3.5.3, this contribution to $D_{\sigma}$ has $L_{2}$ kernels and the kernels converge to limits as $\sigma \rightarrow \infty$. There is a similar convergence for the kernel of $\Gamma Q_{1 \varrho \sigma}$ (Lemma 1.2). Thus the contribution to $C_{\sigma}^{\prime}$ coming from $\Lambda_{3 / 4}$ has an $L_{2}$ kernel and the kernel converges in $L_{2}$ as $\sigma \rightarrow \infty$. This last statement applies to $C_{\sigma}$ also because on $\Lambda_{3 / 4}$ the kernels of $C_{\sigma}$ are bounded by the kernels of $C_{\sigma}^{\prime}$. This is based upon the fact that

$$
\left(\omega^{\prime}+\omega^{\prime \prime}+\omega_{1}+\omega_{2}\right)^{-1} \leqq \text { const. }\left(\omega^{\prime}+\omega^{\prime \prime}-\mu(l)-i\right)^{-1}
$$

on $\Lambda_{3 / 4}$.

Next we consider the contribution to the kernels of $-C_{\sigma}^{\prime}-C_{\sigma}$ coming from $\sim \Lambda_{3 / 4}$. We use the identity

$$
\begin{aligned}
& \left|\left(\omega^{\prime}+\omega^{\prime \prime}+\omega_{1}+\omega_{2}\right)^{-1}\left(\omega_{1}+\omega_{2}+\mu\right)^{-1}-\left(\omega^{\prime}+\omega^{\prime \prime}-\mu-i\right)^{-1}\left(\omega_{1}+\omega_{2}+\mu\right)^{-1}\right| \\
& \quad=\left|\left(\omega^{\prime}+\omega^{\prime \prime}+\omega_{1}+\omega_{2}\right)^{-1}\left(\omega^{\prime}+\omega^{\prime \prime}-\mu-i\right)^{-1}\left(1+i\left(\omega_{1}+\omega_{2}+\mu\right)^{-1}\right)\right| \\
& \quad \leqq \text { const. }\left(\omega^{\prime}+\omega^{\prime \prime}\right)^{-1-2 \varepsilon}\left(\omega_{1}+\omega_{2}\right)^{-(1 / 2)-\varepsilon}
\end{aligned}
$$


on $\sim \Lambda_{3 / 4}$, where $\varepsilon=1 / 12$. Thus these parts of the kernels are bounded by a multiple of

$$
\begin{gathered}
\int\left(\omega^{\prime}+\omega^{\prime \prime}\right)^{-1-2 \varepsilon}\left[\left|\tilde{q}_{1}\left(p^{\prime}, p^{\prime \prime}, k\right)\right|+\left|\tilde{q}_{2}\left(p^{\prime}, p^{\prime \prime}, k\right)\right|\right]\left|\tilde{q}_{2}\left(p^{\prime}, p^{\prime \prime}, l\right)\right| \\
\left(\omega_{1}+\omega_{2}\right)^{-(1 / 2)-\varepsilon}\left|\tilde{q}_{1}\left(p_{1}, p_{2}, l\right)\right| \mid d p^{\prime} d p^{\prime \prime} d l .
\end{gathered}
$$

The function above is in $L_{2}$ and in fact its $L_{2}$ norm is bounded by

$$
\begin{aligned}
&\left\|\left(\omega^{\prime}+\omega^{\prime \prime}\right)^{-(1 / 2)-\varepsilon}\left[\left|\tilde{q}_{1}\right|+\left|\tilde{q}_{2}\right|\right]\right\|_{2}\left\|\left(\omega^{\prime}+\omega^{\prime \prime}\right)^{-(1 / 2)-\varepsilon} \tilde{q}_{2}\right\|_{2} \times \\
& \times\left\|\left(\omega_{1}+\omega_{2}\right)^{-(1 / 2)-\varepsilon} \tilde{q}_{1}\right\|_{2} .
\end{aligned}
$$

The kernels of $-C_{\sigma}^{\prime}-C_{\sigma}$ converge pointwise (for fixed $p_{1}, p_{2}, k$ ) and by the Lebesgue bounded convergence theorem, they converge in $L_{2}$. The lemma is proved.

\subsection{The Definition of $H_{1}$}

It is now easy to prove the main result of this section.

Theorem 3.7.1. Let $\varphi \in \mathscr{D}_{T} \cap \mathscr{D}_{H_{0}^{2}}$ and let

$$
\psi_{\sigma}=T_{\varrho \sigma} \varphi ; \quad \psi=T_{\varrho} \varphi .
$$

As $\sigma \rightarrow \infty$, the limit of $H_{1 \sigma} \psi_{\sigma}$ exists. We call this limit $H_{1} \psi$, so

$$
H_{1} \psi=\lim H_{1 \sigma} \psi_{\sigma} .
$$

This equation defines $H_{1}$ as a symmetric operator with a dense domain $\mathscr{D}_{H_{1}}$.

Proof. We need (3.3.1) and (3.3.2). The formal proof we gave in Sec. 3.3 involved rearrangement of the power series. By Sec. 2 the power series converge absolutely. Thus the rearrangement is justified and the proof of these two formulas is rigorous. We add (3.2.4), (3.3.1) and (3.3.2) to obtain

where

$$
H_{1 \sigma} \psi_{\sigma}=T_{1 \varrho \sigma}: R(\sigma) T_{2 \varrho \sigma}:+T_{\varrho \sigma} H_{0}
$$

$$
\begin{aligned}
R(\sigma)= & Q_{\varrho \sigma}+P(\sigma)+\widetilde{P}(\sigma)-\left[H_{0}, \Gamma Q_{2 \varrho \sigma}\right]+Q_{2 \varrho \sigma} \\
= & {\left[V_{1 \sigma}-Q_{1 \varrho \sigma}-\left[H_{0}, \Gamma Q_{2 \varrho \sigma}\right]\right]+} \\
& +\left[-V_{1 \sigma}-0-\Gamma Q_{1 \varrho \sigma}-Q_{3 \varrho \sigma}\right]+ \\
& +\left[-V_{1 \sigma}-0-\Gamma Q_{\varrho \sigma}-V_{1 \sigma}-0-\Gamma Q_{\varrho \sigma}\right]+ \\
& +\left[-V_{1 \sigma}-0-\left(\Gamma Q_{1 \varrho \sigma}+\Gamma Q_{2 \varrho \sigma}\right)+\Delta_{\sigma}\right]+ \\
& +\left[-V_{1 \sigma}-\bigcirc-\Gamma Q_{3 \varrho \sigma}+C_{\sigma}\right]+ \\
& +\left[-V_{1 \sigma}-0-\Gamma Q_{1 \varrho \sigma}+c_{\sigma} I\right]+ \\
& +\left[2^{-1} V_{1 \sigma}-: \Gamma Q_{\varrho \sigma}{ }^{2}:-A_{\sigma}-B_{\sigma}\right]+ \\
& +\left[-\Delta_{\sigma}-0-\Gamma Q_{\varrho \sigma}-C_{\sigma}+A_{\sigma}\right]+ \\
& +\left[2^{-1} \Delta_{\sigma}-0-: \Gamma Q^{2}{ }_{\varrho \sigma}:+B_{\sigma}\right]+ \\
& +\left[-6^{-1} V_{1 \sigma} \perp: \Gamma Q_{\varrho \sigma}{ }^{3}:\right] .
\end{aligned}
$$


According to the lemmas of this section, each term in the brackets has a limit as $\sigma \rightarrow \infty$ and the convergence satisfies the hypotheses of Theorem 2.2.2. Thus there is a limit operator $R$ and the pair $R(\sigma)$ and $R$ also satisfy these hypotheses. Thus

$$
\lim _{\sigma}: R(\sigma) T_{2 \varrho \sigma}: \varphi=: R T_{\mathbf{2} \sigma}: \varphi
$$

and since the convergence takes place in a subspace with a bounded number of particles, we have

$$
\lim _{\sigma} T_{1 \varrho \sigma}: R(\sigma) T_{2 \varrho \sigma}: \varphi=T_{1 \varrho}: R T_{2 \varrho}: \varphi
$$

by Theorem 2.3.2. Again by Theorem 2.3.2

Thus the limit

$$
\lim T_{\varrho \sigma} H_{0} \varphi=T_{\varrho} H_{0} \varphi \text {. }
$$

$$
H_{1} \psi=\lim _{\sigma} H_{1 \sigma} \psi_{\sigma}
$$

exists. If $\psi=0$ then $\varphi=0$ since $T_{\varrho}$ is invertible (Theorem 2.3.1). Thus $\psi_{\sigma}=T_{\varrho \sigma} \varphi=0$, and so $H_{1} \psi=\lim _{\sigma} 0=0$. It follows that $H_{1}$ is an operator. $H_{1}$ is symmetric because each $H_{1 \sigma}$ is symmetric. $H_{1}$ is densely defined because of Theorem 2.3.3.

Remark. Theorem 3.7.1 is independent of a finite change in the renormalization constants. Let $R$ be the operator

$$
R=a \int: \Phi(x)^{2}: h(x)^{2} d x+b I
$$

(with $a$ and $b$ finite numbers). What we are asserting is that if $H_{1}$ is replaced by $H_{1}+R$ and $H_{1 \sigma}$ is replaced by $H_{1 \sigma}+R$ then the theorem is still true. To see this we note that the first term in $R$ has an $L_{2}$ kernel when expressed in momentum space. Thus the domain of $R$ contains the domain of $N$ and this contains the range of $T_{\boldsymbol{e}}$ and $T_{\varrho \sigma}$ for each $\sigma$. This proves the assertion.

\section{The Definition of $\boldsymbol{H}_{\mathrm{ren}}$ (Conclusion)}

We complete the definition of $H_{\text {ren }}$ as a symmetric bilinear form on $D_{H_{1}} \times D_{H_{1}}$. Since

$$
H_{\text {ren }}=H_{1}+V_{2}
$$

it only remains to define $V_{2}$ as a bilinear form. This can be done quite easily. After multiplying and dividing contracted fermion variables by $\omega^{\tau}$ as in Lemma 3.4.1, $4^{-1}<\tau<2^{-1}$, we can replace $V_{2}$ by an operator $\widetilde{V}_{2}$ with an $L_{2}$ kernel. The desired result then follows from the trivial Lemma 1.1. We do not follow this method, however. Instead we prove a sharper result. We prove that $V_{2}$ and $V_{2 \sigma}$ define bilinear forms which are infinitely small with respect to $F_{\tau}$, uniformly in $\sigma$, for $2^{-1}<\tau<1$. Thus we prove that for each $\varepsilon>0$ there is a $K=K(\varepsilon)$ which does not depend on $\sigma$ such that

$$
\left|\left(\psi, V_{\mathbf{2}} \psi\right)\right|+\left|\left(\psi, V_{\mathbf{2} \sigma} \psi\right)\right| \leqq \varepsilon\left(\psi,\left(F_{\tau}+K I\right) \psi\right)
$$


We also prove that

$$
\lim _{\sigma}\left(\psi_{1}, V_{2 \sigma} \psi_{2}\right)=\left(\psi_{1}, V_{2} \psi_{2}\right)
$$

for $\psi_{1}, \psi_{2}$ in the domain of $F_{\tau}^{1 / 2}$. These two facts combined with Theorems 2.3.4 and 3.7.1 give us the next theorem, which is a summary of the main results of this paper.

Theorem 4.1. $H_{\text {ren }}$ is a symmetric bilinear form defined on $D_{H_{1}} \times D_{H_{1}}$. $H_{\mathrm{ren}}$ is a limit of the bilinear forms $H_{\mathrm{ren}}(\sigma)=H_{1 \sigma}+V_{2 \sigma}$ in the following sense. If

then $\psi_{\sigma} \rightarrow \psi=T_{\varrho} \varphi$ and

$$
\psi_{\sigma}=T_{\varrho \sigma} \varphi, \quad \varphi \in \mathscr{D}_{T} \cap \mathscr{D}_{H_{0}}{ }^{2}
$$

$$
\left(\psi_{\sigma}, H_{\text {ren }}(\sigma) \psi_{\sigma}\right) \rightarrow\left(\psi, H_{\text {ren }} \psi\right) .
$$

Remark. This theorem is independent of a finite change in the renormalization constants.

Proof of (4.1) and (4.2). $V_{2}$ contains four terms corresponding to emission and absorption of mesons by nucleons and antinucleons. These terms are all similar and we consider only one, corresponding to emission of a meson by a nucleon. This term has the form

$$
R=\int r\left(p_{1}, p_{2}, k\right) a^{*}(k) b^{*}\left(p_{1}\right) b\left(p_{2}\right) d p_{1} d p_{2} d k .
$$

We assert that

$$
\mu^{-\tau / 2} \omega_{2}^{-\tau / 2} r \in L_{2} .
$$

Then $V_{2}$ and $V_{2 \sigma}$ are infinitely small with respect to $F_{\tau}$ for any given $\sigma$, by the corollary to Theorem 2.4.3. From the theorem itself we conclude tht

$$
\left|\left(\psi,\left(V_{2 \sigma}-V_{2 \sigma^{\prime}}\right) \psi\right)\right| \leqq o(1)\left(\psi,\left(F_{\tau}+K I\right) \psi\right)
$$

where $o(1) \rightarrow 0$ as $\sigma^{\prime} \rightarrow \sigma$ and

$$
\left|\left(\psi,\left(V_{2}-V_{2 \sigma}\right) \psi\right)\right| \leqq o(1)\left(\psi,\left(F_{\tau}+K I\right) \psi\right)
$$

where $o(1) \rightarrow 0$ as $\sigma \rightarrow \infty$. The second statement implies (4.2), and the two of them together imply that the $K$ in (4.1) can be chosen independently of $\sigma$.

Our kernel $r$ is given by the formula

where

$$
r=\hat{h}\left(k+p_{1}-p_{2}\right) \mu^{-1 / 2} T\left(p_{1} \cdot p_{2}\right)
$$

$T\left(p_{1}, p_{2}\right)=\left(\omega_{1} \omega_{2}\right)^{-1 / 2}\left[\left(\left(\omega_{1}+p_{1}\right)\left(\omega_{2}-p_{2}\right)\right)^{1 / 2}+\left(\left(\omega_{1}-p_{1}\right)\left(\omega_{2}+p_{2}\right)\right)^{1 / 2}\right]$. We note that

$$
\left|T\left(p_{1}, p_{2}\right)\right| \leqq\left(\omega_{1}^{-1}+\omega_{2}^{-1}\right)
$$

in the first and third quadrants of the $p_{1}, p_{2}$ plane

In the region $2|\xi|<|\eta|, T$ is bounded by const $(1+|\eta|)^{-1}$ and (4.3) is bounded by

$$
\text { const. } \mu^{-(\tau+1) / 2} \omega^{-\tau / 2}(1+|\xi+k|)^{-1}(1+|\eta|)^{-1}
$$

which is in $L_{2}$ for any $\tau>0$. The contribution to the $L_{2}$ norm of (4.3) 
which comes from the complementary region $2|\xi| \geqq|\eta|$ is bounded by

$$
\begin{aligned}
& \text { const. }\left(\int_{2|\xi| \geqq|\eta|} \mu^{-\tau-1} \omega_{2}^{-\tau}(1+|\xi+k|)^{-2} d k d \xi d p_{2}\right)^{1 / 2} \leqq \\
& \leqq \text { const. }\left(\int \mu^{-\tau-1}(1+|\xi|)^{-\tau+1}(1+|\xi+k|)^{-2} d k d \xi\right)^{1 / 2}
\end{aligned}
$$

which is finite for $\tau>1 / 2$.

Let $\Xi$ be the region

and let

$$
|\eta| \leqq|\xi|^{2(1-\tau)}
$$

$$
\begin{aligned}
Q_{2}(\Xi) & =\int q_{2} d p_{1} d p_{2} d l \\
Q_{2}(\sim \Xi) & =Q_{2}-Q_{2}(\Xi) .
\end{aligned}
$$

Then $Q_{2}(\Xi)$ is the only part of $Q_{2}$ which contributes to the infinities, and $Q_{2}(\Xi)$ could have replaced $Q_{2}$ in the definition of $Q$. If this were done, the term $Q_{2}(\sim \Xi)$ would be included in the small bilinear form $V_{2}$ rather than in the operator $H_{1}$. To show that this is possible we prove.

Proposition 4.1. Let $2^{-1}<\tau<1 . Q_{2}(\sim \Xi)$ and $Q_{2 \sigma}(\sim \Xi)$ define bilinear forms on $D_{H_{1}}$ which are infinitely small with respect to $F_{\tau}$, uniformly with respect to $\sigma$. On the domain of $F_{\tau}{ }^{1 / 2}, Q_{2 \sigma}(\sim \Xi)$ converges to $Q_{2}(\sim \Xi)$ as $\sigma \rightarrow \infty$.

Proof. This will follow from Theorem 2.4.3 and its corollary. As in the proof of (4.1) and (4.2) we have only to show that

or that

$$
\omega_{1}^{-\tau / 2} \mu^{-\tau / 2}\left|q_{2}\right| \in L_{2}
$$

$$
\int_{\Xi}(|\eta-k|+1)^{-2} \omega_{1}^{-\tau} \mu^{-1-\tau} d p_{1} d \eta d k<\infty .
$$

However the $p_{1}$ integration can be bounded by

$$
\int_{0}^{K|\eta|^{1 / 2(1-\tau)}} \omega_{1}^{-\tau} d p_{1} \leqq \text { const. }\left(1+|\eta|^{1 / 2}\right),
$$

where $K$ is some constant, and so the previous integral is finite as required.

\section{Appendix}

For the reader's convenience we give here a proof that our expression in Sec. 1 for the free fermion field can be obtained from a more customary expression in which the Fock space consists of spinor valued functions. Our notation comes primarily from [4].

Let $e_{0}$ and $e_{1}$ be a base for $R^{2}$. We choose a Lorentz inner product $(.,$.$) in R^{2}$ so that

Let

$$
\left(e_{0}, e_{0}\right)=-1, \quad\left(e_{1}, e_{1}\right)=+1 .
$$

$$
\gamma_{0}=\gamma\left(e_{0}\right)=\left(\begin{array}{ll}
0 & i \\
i & 0
\end{array}\right), \quad \gamma_{1}=\gamma\left(e_{1}\right)=\left(\begin{array}{rr}
0 & i \\
-i & 0
\end{array}\right)
$$


Let $\gamma^{0}=-\gamma_{0}, \gamma^{1}=\gamma_{1}$ and if $u=u^{\mu} e_{\mu}$ (summation convention) let

We compute directly

$$
\gamma(u)=u^{\mu} \gamma_{\mu}=\left(\begin{array}{cc}
0 & i\left(u^{0}+u^{1}\right) \\
i\left(u^{0}-u^{1}\right) & 0
\end{array}\right)
$$

$$
\{\gamma(u), \gamma(v)\}=2(u, v)=2\left(-u^{0} v^{0}+u^{1} v^{1}\right)
$$

The Dirac equation is

$$
\left(\gamma^{\mu}\left(\partial / \partial x^{\mu}\right)+\omega_{0}\right) \psi=0
$$

where $\psi$ is a function with values in $C^{2}$, complex two space. If $\psi(x)$ $=u e^{i p x}$ is a solution, we must have

$$
0=\left(i \gamma^{\mu} p_{\mu}+\omega_{0}\right) u=\left(i \gamma(p)+\omega_{0}\right) u
$$

and hence $(p, p)+\omega_{0}^{2}=0$ and if $p^{0}>0$ then $u$ must be a multiple of

$$
\nu(p)=\left(\begin{array}{l}
\left(p^{0}+p^{1}\right)^{1 / 2} \\
\left(p^{0}-p^{1}\right)^{1 / 2}
\end{array}\right)=\left(\begin{array}{l}
\nu^{1}(p) \\
\nu^{2}(p)
\end{array}\right) \text {. }
$$

Let $\langle$,$\rangle be the hermitian inner product in C^{2}$ (antilinear in the first variable), and let $\beta$ be the antilinear map from $C^{2}$ to its dual given by

$$
\begin{aligned}
(\beta u)(v) & =\left\langle\left(-i \gamma_{0}\right) u, v\right\rangle \\
& =\bar{u}^{1} v^{2}+\bar{u}^{2} v^{1} .
\end{aligned}
$$

We set $\beta u=u^{\dagger}$ and we define $u^{c}$, the charge conjugation of $u$, to be the complex conjugate of the vector

$$
\left(\begin{array}{rr}
1 & 0 \\
0 & -1
\end{array}\right) u \text {. }
$$

We note that $\nu^{\dagger} v=2 \omega_{0}=-v^{c \dagger} \nu^{c}$ and $v^{\dagger} v^{c}=0=v^{c \dagger} v$ if $p^{0}>0$. Also

if $p^{0}>0$.

$$
\nu^{c}(p)=\left(\begin{array}{r}
\left(p^{0}+p^{1}\right)^{1 / 2} \\
-\left(p^{0}-p^{1}\right)^{1 / 2}
\end{array}\right)
$$

If $f \in \mathbf{D}^{+}$then

$$
\psi(p)=f\left(p^{1}\right) \nu(p)
$$

is the expression in Fourier Transform space of a solution $\tilde{\psi}(x)$ of the Dirac equation. We introduce the norms

$$
\begin{aligned}
\|\tilde{\psi}(x)\|^{2}=\|\psi(p)\|^{2} & =\left(2 \omega_{0}\right)^{-1} \int_{p^{0}>0} \psi^{\dagger} \psi d \Omega(p) \\
& =\left(2 \omega_{0}\right)^{-1} \int_{p^{0}>0}|f|^{2} \nu^{\dagger} v d \Omega(p)=\int \omega^{-1}|f|^{2} d p^{1}=\|f\|_{D^{+}}^{2},
\end{aligned}
$$

where $d \Omega$ is the Lorentz invariant measure on the hyperboloid $(p, p)+\omega_{0}^{2}=0$.

The equation (A.1) thus defines a unitary equivalence between $\mathbf{D}^{+}$ and the conventional single particle space of positive energy solutions of the Dirac equation. The isomorphism extends to tensor products and provides an isomorphism of $\mathbf{F}$ with a Fock space in which the nucleons are spinor valued functions. Under this isomorphism, the operators 
$b\left(p^{1}\right)$ and $b^{*}\left(p^{1}\right)$ are mapped onto the operators

and

$$
b\left(p^{1}\right) v(p)^{\dagger}=\tilde{b}(p)
$$

$$
b^{*}\left(p^{1}\right) v(p)=\tilde{b}^{*}(p)
$$

Let $\tilde{b}^{\prime}$ and $\tilde{b}^{\prime *}$ be the antinucleon annihilation and creation operators, acting on the Fock space of spinor valued antinucleon functions. The field $\Psi$ is given by the expression (cf. [4, p. 156]; for simplicity we have set $\left.x^{0}=0\right)$.

$$
\int_{p^{0}=\omega}\left(e^{i p x} v^{\alpha}(p) \tilde{b}(p)+e^{-i p x} v^{c \alpha}(p) \tilde{b}^{\prime *}(p)\right)\left(p^{0}\right)^{-1 / 2} d p^{1} .
$$

This integral corresponds to the definition of $\Psi^{\alpha}(x)$ given in Sec. 1 .

\section{References}

1. Berezin, F.: On the Lee model. Mat. Sbornik 60, 425-446 (1963).

2. Friedrichs, K.: Perturbation of spectra in Hilbert space. Am. Math. Soc. Providence, 1965.

3. GLImM, J.: The Schrödinger equation for quatum fields with nonlinear nonlocal scattering. Commun. Math. Phys. 2, 271-300 (1963).

4. Kastler, D.: Introduction a l'electrodynamique Quantique. Paris: Dunod 1961.

5. Kristensen, P., L. Mejlbo, and E. Poulsen: Tempered distributions in infinitely many dimensions II. Math. Scand. 14, 129-150 (1964).

6. LANFORD, O.: Construction of quantum fields interacting by a cutoff Yukawa coupling. Princeton University Thesis 1966.

7. Nelson, E.: A quartic interaction in two dimensions. In: Mathematical theory of elementary particles, ed. by R. Goodman and I. Segat, pp. 69-73. M.I.T. Press 1965.

8. Streater, R., and A. Wightman: PCT, spin and statistics and all that. New York: Benjamin Inc. 1964.

9. Wightman, A.: Introduction to some aspects of the relativistic dynamics of quantized fields. Institute des Hautes Etudes Scientifiques, Bures-sur-Yvette (Revised notes for lectures at the French summer school of theoretical physics, Cargese, Corsica, July 1964). 